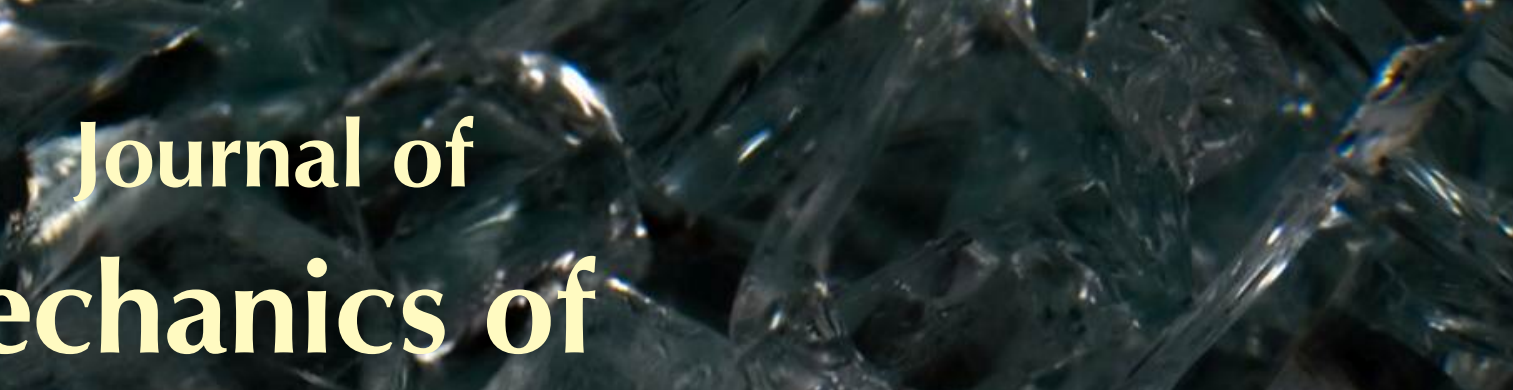

Materials and Structures

Mechanics of $7:$
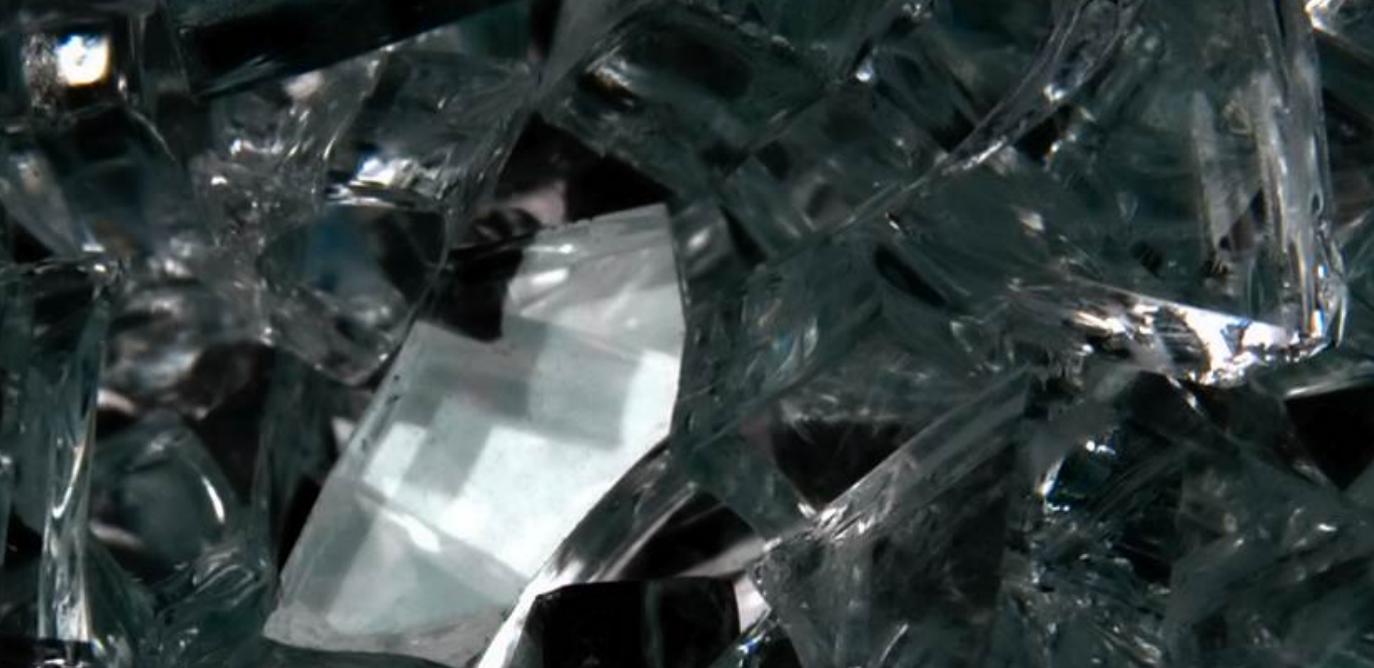

की

A DIFHUSE COHESIVE ENERGY APPROȦCH TO FRACTỨRE

AND PLASTICITY: THE ONE-DIMIENSIONAL CASE

Gianpietro Del Piero, Giovanni Lancioni and Riccardo March :- -

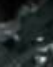

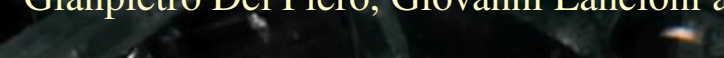

2

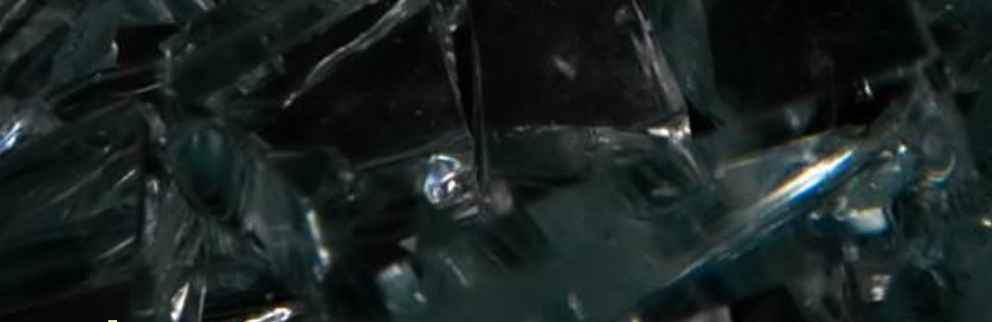

Volume 8, No. $2-4 \quad \ldots 2$

- April-June 2013

N.

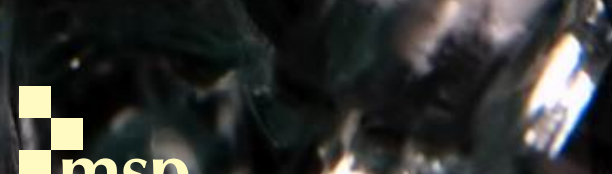




\title{
A DIFFUSE COHESIVE ENERGY APPROACH TO FRACTURE AND PLASTICITY: THE ONE-DIMENSIONAL CASE
}

\author{
Gianpietro Del Piero, Giovanni Lancioni And Riccardo March
}

\begin{abstract}
In the fracture model presented in this paper, the basic assumption is that the energy is the sum of two terms, one elastic and one cohesive, depending on the elastic and inelastic part of the deformation, respectively. Two variants are examined: a local model, and a nonlocal model obtained by adding a gradient term to the cohesive energy. While the local model only applies to materials which obey Drucker's postulate and only predicts catastrophic failure, the nonlocal model describes the softening regime and predicts two collapse mechanisms, one for brittle fracture and one for ductile fracture.

In its nonlocal version, the model has two main advantages over the models existing in the literature. The first is that the basic elements of the theory (the yield function, hardening rule, and evolution laws) are not assumed, but are determined as necessary conditions for the existence of solutions in incremental energy minimization. This reduces to a minimum the number of independent assumptions required to construct the model. The second advantage is that, with appropriate choices of the analytical shape of the cohesive energy, it becomes possible to reproduce, with surprising accuracy, a large variety of observed experimental responses. In all cases, the model provides a description of the entire evolution, from the initial elastic regime to final rupture.
\end{abstract}

\section{Introduction}

Since the pioneering paper [Francfort and Marigo 1998], the variational approach to fracture has been the object of intense research. A reason for this is the availability of numerical instruments supporting the theory, which are capable of solving practical problems in fracture mechanics. One such instrument is the regularization technique of Ambrosio and Tortorelli [1990], initially conceived for the image segmentation problem [Mumford and Shah 1989], and applied to fracture problems by Bourdin, Francfort and Marigo [2000]. The regularization consists in approximating the energy of a fractured body, which by its own nature is defined on discontinuous deformations with bounded variation, by a family of functionals defined in a Sobolev space. This makes it possible to solve fracture problems using standard finite element techniques.

The original paper [Francfort and Marigo 1998] and the subsequent developments were based on the brittle fracture model of Griffith [1920], in which the presence of singular discontinuity surfaces for the displacement is allowed at the interior of an elastic body. In fact, long before the appearance of [Francfort and Marigo 1998], more sophisticated models, based on Barenblatt's cohesive fracture hypothesis [Barenblatt 1962], were used in fracture mechanics. They were particularly efficient in situations in which fracture is preceded by a regime of large inelastic deformation, like in the rupture of concrete and other nonmetallic materials [Hillerborg 1991; Carpinteri and Massabò 1997]. In this respect, a fundamental

Keywords: nonlocal plasticity, strain localization, variational fracture, ductile fracture, incremental energy minimization. 
role was played by the cohesive crack model of [Hillerborg et al. 1976]. The development of the theories of rupture for concrete over the following decades is documented in the review papers [Carpinteri 1982; Bažant and Chen 1997; Bažant and Jirásek 2002; Bažant and Le 2009].

With the cohesive energy model, two basic aspects of energy minimization became evident. The first is the relevance of local, versus global, minimization. This point has received significant attention in the recent literature [Dal Maso and Toader 2002; Marigo and Truskinovsky 2004], and is now generally accepted. The second aspect is the relevance of the role played by energy dissipation. Indeed, with a minimization constrained by a dissipation inequality it is possible to reproduce some sophisticated aspects of material response such as, for instance, elastic unloading in plasticity [Del Piero and Truskinovsky 2009].

The present paper originates from a reflection on similarities and differences between the cohesive energy scheme and the Ambrosio-Tortorelli approximation. For both, the energy functional depends on the elastic deformation plus a second variable which provides a regularized representation of fracture. But, while the cohesive energy is usually assumed to be a surface energy, the regularizing term in [Ambrosio and Tortorelli 1990] is a volume integral.

The question naturally arises, of whether or not it is convenient to assume a cohesive energy concentrated on singular surfaces. In ductile materials, before rupture, the appearance of a process zone is observed, in which the material becomes weaker and more deformable. This phenomenon can be described by damage [Babadjian 2011] or plasticity [Dal Maso and Toader 2010] theories. Cohesive energies diffused over the volume were recently considered in the modeling of brittle fracture [Volokh 2004] and damage [Benallal and Marigo 2007; Pham et al. 2011], and in the variational theory of fracture [Freddi and Royer-Carfagni 2010]. In particular, [Pham and Marigo 2010a] deals with a model of energy minimization, in which rupture is preceded by progressive damage. In it, the one-dimensional energy functional has the form

$$
\int_{0}^{l}\left(w\left(u^{\prime}(x), \gamma(x)\right)+\theta(\gamma(x))\right) d x
$$

where $w$ is the bulk energy density, $u^{\prime}$ is the axial deformation, and $\gamma$ is an internal variable representing the intensity of damage. In the present paper we consider the special case

$$
\int_{0}^{l}(w(\epsilon(x))+\theta(\gamma(x))) d x
$$

in which $\epsilon(x)=u^{\prime}(x)-\gamma(x)$ is the elastic part of the deformation. Of the two energy densities $w$ and $\theta$, the first is assumed to be reversible and the second is assumed to be totally dissipated. By assuming $w$ independent of $\gamma$, we purposely renounce any description of damage. Though our initial purpose was to construct something more general than a plasticity theory, to our surprise we realized that, as we shall see below, the energy (1-2) provides exactly the classical incremental theory of plasticity exposed, for example, in [Hill 1950].

A weak point of the model is that it fails to describe the strain-softening response which, as wellknown from the literature, is associated with localization of the plastic deformation. These effects can be captured by adding a nonlocal term of the gradient type

$$
\int_{0}^{l}(w(\epsilon(x))+\theta(\gamma(x))) d x+\frac{1}{2} \alpha \int_{0}^{l} \gamma^{\prime 2}(x) d x,
$$


which successfully captures some specific effects of material response in a large variety of models, like the van der Waals model for capillarity [van der Waals 1893; Casal 1972], the Cahn-Hilliard model for phase transition [Cahn and Hilliard 1958], Mumford-Shah image segmentation theory [Mumford and Shah 1989], some theories of liquid crystals [Frank 1958; Ericksen 1990], and some numerical models for the rupture of concrete [Borst and Pamin 1996]. A nonlocal term was also used in [Truskinovsky 1996] to construct a cohesive zone model starting from the energy of interatomic attraction (Lennard-Jones potential).

The addition of a gradient term renders the expression (1-3) of the energy similar to the AmbrosioTortorelli functional

$$
\int_{0}^{l}\left(s^{2}(x)+\kappa_{\alpha}\right) w\left(u^{\prime}(x)\right) d x+\frac{1}{2} \alpha \int_{0}^{l}\left(s^{\prime 2}(x)+\frac{c}{\alpha^{2}}(1-s(x))^{2}\right) d x .
$$

Indeed, in both cases the total energy is the sum of a basic energy and a perturbing gradient term, and in both cases the deformation is accompanied by a second independent variable. The main difference is that in (1-3) the second variable is the inelastic part of the deformation, while in (1-4) the scalar field $s \in[0,1]$ measures the fracture intensity in the regularized model. Also different is the nature of the smallness parameter $\alpha$. In (1-4), $\alpha$ is the coefficient of a singular perturbation, and what is important is the asymptotic behavior of the solution when $\alpha \rightarrow 0$. In fracture theory, $\alpha$ is a fixed material constant, usually interpreted as an internal length determined by the microscopic structure of the material [Bažant and Pijaudier-Cabot 1989; Bažant and Jirásek 2002; Pham et al. 2011].

The value of $\alpha$ strongly influences the material's response. Indeed, the ratio between internal length and the length $l$ of the bar is an indicator of the brittleness of the fracture. A reduction of $\alpha$ has the same effect as an increase of $l$, that is, a transition from rupture preceded by a long regime of gradual weakening to sudden catastrophic failure. For fixed $\alpha$ and varying $l$, this is the well-known size effect of fracture mechanics.

The requirement of nonnegativeness of the first variation leads to Kuhn-Tucker conditions very similar, and sometimes identical, to the governing equations of the gradient theories of plasticity [Aifantis 1992; Fleck and Hutchinson 2001; Gurtin and Anand 2005] and damage [Benallal and Marigo 2007; Pham and Marigo 2010b; Pham et al. 2011], which, starting from the pioneering paper [Aifantis 1984], have been developed by several authors in the last two decades. In particular, the expression of the first variation of the energy given in Section 3.2 is the one-dimensional counterpart of the expression of the virtual power currently assumed in the theories of strain-gradient plasticity [Gurtin 2003; Gudmundson 2004; Gurtin and Anand 2005].

An accurate review, analysis, and comparison of such theories can be found in [Jirásek and Rolshoven 2009a; 2009b]. Most theories include a number of assumptions which fix the basic physical aspects of plastic response:

- the stress-strain elastic law,

- the hardening rule,

- the evolution laws,

- the loading-unloading conditions,

- the yield function. 
The underlying continuum can be either a classical or a generalized continuum. In a classical continuum the plastic deformation is regarded as a state variable, the Cauchy stress is the unique stress measure, and supplementary generalized forces are defined as the conjugates of the gradients of the plastic deformation. In a generalized continuum, higher-order stresses appear as conjugates of the higher-order deformation gradients.

The two cases are analyzed in [Jirásek and Rolshoven 2009b] and [2009a], respectively. The number and variety of theories reported therein shows by itself that there is yet no agreement about the choice of a fully satisfactory model. The present paper has the ambition of presenting a mathematically sound model, based on very simple assumptions, and providing a very flexible description of the entire evolution from the initial elastic regime to final rupture.

The basic assumptions are the forms of the elastic energy and of the dissipative cohesive energy. Proceeding by incremental energy minimization, all classical hypotheses listed above are obtained as necessary conditions for a minimum. The analytical forms of the functions defined in the above hypotheses are already contained in the assumed form of the cohesive energy. In particular, the dissipative character assumed for the cohesive energy provides the basis for the description of elastic unloading, and the shape of the cohesive energy can be chosen to reproduce a large variety of responses, hardening or softening, and with the plastic strain diffusing over the body or localizing on small regions.

Surface cohesive energies have proved to be an efficient tool for formulating and solving fracture problems. Here we show that volume cohesive energies can be a very natural way for describing fracture as a terminal event in an evolution involving plasticity or damage. Of course, there is no claim of generality for the model presented here. It can be generalized in several directions. We just like to compare the simplicity of the starting assumptions and of the incremental minimizing procedure, with the richness of the responses that can be obtained by acting on the analytical shape of the cohesive energy.

Our analysis is restricted to the one-dimensional case. This allows us to get the basic results avoiding a number of technical complications, at the cost of neglecting some important aspects congenital to higher dimension. The paper is divided into four sections. After the present introduction, Sections 2 and 3 deal with the local and nonlocal models, respectively, and Section 4 reports some numerical simulations on the nonlocal model and compares them with experiments. The local model is introduced in Section 2.1, and in Section 2.2 the equilibrium configurations are identified with the stationary points of the energy (1-2). Due to the unilateral character of the dissipation constraint, the Euler equation is replaced by an inequality. This inequality provides an upper bound for the axial force, depending on the current value of the plastic deformation. In this way, the yield condition is obtained.

The minimum properties of the stationary points are investigated in Section 2.3. The following subsections deal with the quasistatic evolution of the inelastic deformation under varying load, along a path made of energy minimizers. For simplicity, we only consider a bar under a hard device, for which the load consists of displacements applied at the endpoints. Section 2.4 is devoted to incremental energy minimization. In a first-order approximation, the minimization consists in determining the direction of steepest descent under the given load increment. The result is that along that direction the inelastic deformation does not change in the elastic regime, that is, when the yield condition is satisfied as a strict inequality. On the contrary, in the inelastic regime the inelastic strain rate remains unspecified.

For its determination, a second-order minimization is required. In it, some basic properties of classical plasticity, such as the hardening rule, the consistency condition for the inelastic deformation at loading, 
and the property of elastic unloading are obtained as necessary conditions for a minimum. The minimization leads to a first-order differential problem, involving the current values of the inelastic deformation and of the inelastic strain rate. This problem is studied in Section 2.5. In the inelastic regime, at each time $t$ the response is determined by the sign of the second derivative of $\theta$ at the current deformation $\gamma_{t}$ : the response is work-hardening for $\theta^{\prime \prime}\left(\gamma_{t}\right)>0$ and perfectly plastic for $\theta^{\prime \prime}\left(\gamma_{t}\right)=0$. For $\theta^{\prime \prime}\left(\gamma_{t}\right)$ negative, the deformation concentrates at a single point, determining catastrophic rupture.

The local model provides a classical incremental plasticity theory [Hill 1950], obeying Drucker's material stability postulate [Drucker 1952]:

$$
\dot{\sigma}_{t} \dot{\gamma}_{t} \geq 0
$$

which does not allow for a strain-softening response. Within this restricted context, the model correctly describes a plastic response followed by brittle fracture in the sense of Griffith's theory.

The nonlocal model based on the energy (1-3) is discussed in Section 3. Due to the presence of a gradient term, more regularity and supplementary boundary conditions are required. The additional regularity assumptions are made in Section 3.1, and two different options for the supplementary boundary conditions are considered in Section 3.2. The choice of the supplementary boundary conditions is a delicate problem; see, for example, the discussion in [Jirásek and Rolshoven 2009b, §3.4]. Our choice of imposing a null inelastic deformation $\gamma$ at the boundary is motivated by a closer adherence to the constraint devices used in laboratory tests.

The minimum properties of the equilibrium configurations, still identified with the stationary points of the energy, are investigated in Section 3.3. Due to the stabilizing effect of the nonlocal term, the necessary conditions for a minimum are weaker than in the local model. In particular, moderate negative values of $\theta^{\prime \prime}(\gamma(x))$ become acceptable, and this renders admissible a strain-softening response. In the language of [Jirásek and Rolshoven 2009a; 2009b], the nonlocal term acts as a localization limiter, since it opposes the localization of the inelastic deformation.

The quasistatic evolution under a given load process is studied in Section 3.4. A first-order minimization still provides the direction of steepest descent for the energy functional. Along this direction, the inelastic strain rate $\dot{\gamma}_{t}(x)$ is zero in the elastic zone, that is, at points $x$ at which the yield condition is satisfied as a strict inequality. Surprisingly enough, this conclusion is partially contradicted in the second-order minimization, in which nonnull inelastic strain rates are allowed inside the elastic zone, at points sufficiently close to the interface with the inelastic zone.

The second-order minimization provides a set of Kuhn-Tucker conditions, which governs the quasistatic evolution of the inelastic deformation. Due to the presence of the nonlocal terms, closed-form solutions can be found only in some special cases. One of them is the onset of the inelastic regime, studied in detail in Section 3.5. In the determination of the incremental response at the onset, a fundamental role is played by the nondimensional ratio $l / l_{i}$, where

$$
l_{i} \doteq 2 \pi \sqrt{\frac{\alpha}{-\theta^{\prime \prime}(0)}}
$$

is the internal length of the material at the onset. Indeed, the response is work-hardening if $l<l_{i} / 2$ and strain-softening if $l>l_{i} / 2$. Moreover, for $l<l_{i}$ the response is full-size, that is, the inelastic strain rate is diffused over the whole bar, while for $l>l_{i}$ the inelastic strain rate localizes on a region of length $l_{i}$. 
It is possible that catastrophic failure occurs just at the onset of the inelastic regime. This circumstance, which we call totally brittle fracture, depends on a second nondimensional ratio $l / l_{c}$, where

$$
l_{c} \doteq 2 \pi \sqrt{\frac{\alpha}{w^{\prime \prime}\left(\beta_{c}\right)}}
$$

is the characteristic length at the onset and $\beta_{c}$ is the value of the load $\beta$ at the onset. In fact, totally brittle fracture occurs when $l / l_{c}$ is less than a given function of $l / l_{i}$.

A qualitative analysis of the response in the inelastic regime is made in Section 3.6. In it, internal length and characteristic length are considered as functions of the current inelastic and elastic deformation

$$
l_{i t}=2 \pi \sqrt{\frac{\alpha}{-\theta_{t}^{\prime \prime}}}, \quad l_{c t}=2 \pi \sqrt{\frac{\alpha}{w^{\prime \prime}\left(\epsilon_{t}\right)}},
$$

respectively, where $\theta_{t}^{\prime \prime}$ is an average of $\theta^{\prime \prime}\left(\gamma_{t}(x)\right)$ over the bar in the current inelastic deformation $\gamma_{t}$. In this way, by extrapolation of the exact results obtained at the onset, it is possible to obtain an approximate picture of the bar's evolution up to final rupture.

In particular, from this qualitative analysis two fundamental types of evolution emerge. They depend on the convexity-concavity properties of the derivative $\theta^{\prime}$ of the cohesive energy, and determine two very different collapse mechanisms. If $\theta^{\prime}$ is concave, an increasing load produces a progressive localization of the inelastic deformation, and a progressive increase of the negative slope of the force-elongation response curve. In the limit, an extreme localization and an infinite slope of the response curve produce catastrophic failure. On the contrary, if $\theta^{\prime}$ is convex, under increasing load the inelastic deformation diffuses over larger and larger zones, and the negative slope of the curve decreases. In the limit the slope tends to zero, and the bar elongates indefinitely at constant force.

The two collapse mechanisms are typical of materials with crystalline structure and with random structure, respectively. In Section 4 both are investigated by means of numerical simulations, with the purpose of reproducing the response curves of two real materials, concrete and steel. The correspondence of the simulations with the experimental curves is obtained by fitting a very small number of parameters, that is, the material constants necessary to represent the cohesive energy $\theta$ as a piecewise polynomial function. The result is impressive. It shows that an appropriate choice of the expression of the cohesive energy can indeed capture not only the overall behavior, but also many detailed features of the response curve, in the whole evolution from the initial natural state up to rupture.

Roughly, the main results of the present study can be summarized as follows.

- In its nonlocal version, the diffuse cohesive energy model captures both brittle and ductile fracture modes. In brittle fracture the inelastic deformation tends to localize on singular surfaces, and in ductile fracture it tends to spread over the whole bar. Brittle fracture may also take place without any preliminary regime of inelastic deformation.

- A convex cohesive energy produces a full-size work-hardening response. A concave energy may produce a full-size or localized, work-hardening or strain-softening response, depending on the ratio between the current internal length and the length of the bar.

- For $\theta$ concave, a convex $\theta^{\prime}$ produces brittle fracture, and a concave $\theta^{\prime}$ produces ductile fracture. 
- Brittle fracture occurs when the current internal length reaches a critical value depending on the current characteristic length.

Some of these results, reflecting an earlier stage of the present research, were announced in [Del Piero et al. 2012]. But even the present results are not complete, since many problems are left open. Specifically, it seems very difficult to reach the same level of detail in a two or three-dimensional context. Also, a big challenge is how to find general, physically motivated correlations between the convexity-concavity properties of $\theta$ and $\theta^{\prime}$ and the microstructural properties of real materials. This would require a truly interdisciplinary effort, far beyond the domain of classical continuum mechanics.

\section{The local model}

2.1. Basic assumptions. Consider a straight bar of length $l$, homogeneous, with a constant cross-section, subject to prescribed axial displacements at the endpoints

$$
u(0)=0, \quad u(l)=\beta l,
$$

and free of applied loads. In what follows, the term load will be used to denote the relative elongation $\beta$. A deformed configuration is described by the axial displacements $u(x)$ of the points $x$ of the bar's axis, and the derivative $u^{\prime}(x)$ is a measure of the axial deformation at $x$.

We make four basic assumptions. The first is that at every $x$ the deformation can be split into the sum of an elastic part $\epsilon(x)$ and an inelastic part $\gamma(x)$ :

$$
u^{\prime}(x)=\epsilon(x)+\gamma(x), \quad x \in(0, l) .
$$

A pair $(\epsilon, \gamma)$ of functions continuous in $[0, l]$ is a configuration of the bar. By integration, from the boundary conditions (2-1) it follows that

$$
\beta=\bar{\epsilon}+\bar{\gamma},
$$

with

$$
\bar{\epsilon}=\frac{1}{l} \int_{0}^{l} \epsilon(x) d x, \quad \bar{\gamma}=\frac{1}{l} \int_{0}^{l} \gamma(x) d x .
$$

Our second assumption is that the strain energy of the bar has the form

$$
E(\epsilon, \gamma)=\int_{0}^{l}(w(\epsilon(x))+\theta(\gamma(x))) d x,
$$

where $w$ and $\theta$ are the volume densities of the elastic strain energy and of the cohesive energy, respectively. We assume that $w$ is $C^{2}$ and strictly convex, and that

$$
w(0)=w^{\prime}(0)=0 .
$$

Then $w^{\prime}$ is strictly increasing, and

$$
w(\epsilon)>0, \quad \forall \epsilon \neq 0, \quad w^{\prime}(\epsilon)\left\{\begin{array}{ll}
>0, & \forall \epsilon>0, \\
<0, & \forall \epsilon<0,
\end{array} \quad w^{\prime \prime}(\epsilon)>0, \quad \forall \epsilon \in \mathbb{R} .\right.
$$


We also assume that $\theta$ is $C^{2}$ and monotonic increasing, and that

$$
\theta(0)=0, \quad \theta^{\prime}(0)>0, \quad \lim _{\gamma \rightarrow+\infty} \theta(\gamma)<+\infty .
$$

The third assumption is that the elastic part of the deformation is related to the axial force $\sigma$ by the constitutive equation

$$
\sigma=w^{\prime}(\epsilon),
$$

and the fourth assumption is that $w$ can be stored, while $\theta$ is totally dissipated. That is, in every deformation process $t \mapsto\left(\epsilon_{t}, \gamma_{t}\right)$, at every $x$ the cohesive power is nonnegative:

$$
\theta^{\prime}\left(\gamma_{t}(x)\right) \dot{\gamma}_{t}(x) \geq 0 \text {. }
$$

Here and in the following, a superimposed dot denotes the right derivative with respect to the parameter $t$. Due to the rate-independent character of the model, $t$ may be any monotonic nondecreasing function of the physical time. In what follows we consider the case of $\theta$ strictly monotonic, for which the dissipation inequality

$$
\dot{\gamma}_{t}(x) \geq 0
$$

holds at all $x$ and for all $t$.

2.2. Equilibrium. For a given configuration $(\epsilon, \gamma)$, a perturbation is a pair $(\delta \epsilon, \delta \gamma)$ such that

$$
\delta \epsilon(x)=\dot{\epsilon}_{t}(x), \quad \delta \gamma(x)=\dot{\gamma}_{t}(x),
$$

in some deformation process with $\left(\epsilon_{t}, \gamma_{t}\right)=(\epsilon, \gamma)$. While $\delta \epsilon$ is unrestricted, $\delta \gamma$ is subject to the condition

$$
\delta \gamma(x) \geq 0, \quad \forall x \in(0, l),
$$

due to the dissipation inequality (2-10). We say that $(\epsilon, \gamma)$ is an equilibrium configuration if the first variation of the energy

$$
\begin{aligned}
\delta E(\epsilon, \gamma, \delta \epsilon, \delta \gamma) & =\lim _{\lambda \rightarrow 0^{+}} \frac{1}{\lambda}(E(\epsilon+\lambda \delta \epsilon, \gamma+\lambda \delta \gamma)-E(\epsilon, \gamma)) \\
& =\int_{0}^{l}\left(w^{\prime}(\epsilon(x)) \delta \epsilon(x)+\theta^{\prime}(\gamma(x)) \delta \gamma(x)\right) d x
\end{aligned}
$$

is nonnegative for all perturbations which preserve the length of the bar

$$
\delta \bar{\epsilon}+\delta \bar{\gamma}=0, \quad \delta \bar{\epsilon}=\frac{1}{l} \int_{0}^{l} \delta \epsilon(x) d x, \quad \delta \bar{\gamma}=\frac{1}{l} \int_{0}^{l} \delta \gamma(x) d x .
$$

In particular, for perturbations with $\delta \gamma=0$ the first variation is

$$
\delta E(\epsilon, \gamma, \delta \epsilon, 0)=\int_{0}^{l} w^{\prime}(\epsilon(x)) \delta \epsilon(x) d x .
$$

By (2-13), $\delta \bar{\epsilon}=0$ for such perturbations. Then, a standard argument of the calculus of variations leads to the conclusion that the first variation is nonnegative if and only if $w^{\prime}(\epsilon(x))$ is a constant. 
By the constitutive equation (2-8), this implies that the axial force $\sigma$ is constant. Moreover, by the strict convexity of $w$, the derivative $w^{\prime}$ is strictly increasing and, therefore, invertible. Then, $\epsilon$ is constant as well:

$$
\epsilon=\left(w^{\prime}\right)^{-1}(\sigma)
$$

By (2-13), for constant $\epsilon$ the first variation reduces to

$$
\delta E(\epsilon, \gamma, \delta \epsilon, \delta \gamma)=\int_{0}^{l}\left(\theta^{\prime}(\gamma(x))-w^{\prime}(\epsilon)\right) \delta \gamma(x) d x,
$$

and because $\delta \gamma(x)$ is arbitrary nonnegative, the condition

$$
w^{\prime}(\epsilon) \leq \theta^{\prime}(\gamma(x)), \quad \forall x \in(0, l),
$$

follows. Conversely, for every configuration $(\epsilon, \gamma)$ obeying this condition the first variation is nonnegative. Therefore, an equilibrium configuration is a configuration $(\epsilon, \gamma)$, with constant $\epsilon$, which satisfies condition (2-15) for all $x$.

An equilibrium configuration is equilibrated with the load $\beta$ given by (2-3). Then inequality (2-15), rewritten in the form

$$
w^{\prime}(\beta-\bar{\gamma}) \leq \theta^{\prime}(\gamma(x)), \quad \forall x \in(0, l),
$$

shows that an equilibrium configuration can be defined alternatively as a pair $(\beta, \gamma)$ which satisfies (2-11) and (2-16). There are many $\gamma$ for which $(\beta, \gamma)$ is an equilibrium configuration. Indeed, by the assumptions made on $w$ and $\theta$, all configurations $(\beta, \gamma)$ with $\bar{\gamma}>\beta$ satisfy inequality (2-16), because the right side of the inequality is nonnegative, while for $\beta<\bar{\gamma}$ the left side is negative.

With every equilibrium configuration $(\beta, \gamma)$ are associated the axial force

$$
\sigma=w^{\prime}(\beta-\bar{\gamma})
$$

and the energy

$$
E^{\mathrm{eq}}(\beta, \gamma) \doteq l w(\beta-\bar{\gamma})+\int_{0}^{l} \theta(\gamma(x)) d x .
$$

The set of all $x$ at which inequality (2-16) is strict is the elastic zone

$$
\mathscr{E}(\beta, \gamma) \doteq\left\{x \in(0, l) \mid w^{\prime}(\beta-\bar{\gamma})<\theta^{\prime}(\gamma(x))\right\}
$$

and the complementary set

$$
\mathscr{g}(\beta, \gamma) \doteq(0, l) \backslash \mathscr{E}(\beta, \gamma)
$$

at which (2-16) is verified as an equality is the inelastic zone. We say that the bar is in the elastic regime if $\mathscr{E}(\beta, \gamma)=(0, l)$, and that it is in the inelastic regime otherwise.

The equilibrium condition (2-16), rewritten in the form

$$
\sigma \leq \theta^{\prime}(\gamma(x)), \quad \forall x \in(0, l),
$$

shows that there is an upper limit for $\sigma$. In the language of plasticity, this inequality is a yield condition, which imposes that the current stress $\sigma$ not be greater than the yield limit $\theta^{\prime}$. The difference $\theta^{\prime}-\sigma$ is the yield function. 
2.3. Energy minimizers. Among all inelastic deformations $\gamma$ equilibrated with a given $\beta$, of interest are those which minimize the energy $E^{\mathrm{eq}}(\beta, \cdot)$. A necessary condition for a minimum at $\gamma$ is that, in the expansion

$$
\begin{aligned}
E^{\mathrm{eq}}(\beta, \gamma+\lambda \delta \gamma)-E^{\mathrm{eq}}(\beta, \gamma)=\lambda \int_{0}^{l}\left(\theta^{\prime}(\gamma(x))\right. & \left.-w^{\prime}(\beta-\bar{\gamma})\right) \delta \gamma(x) d x \\
& +\frac{1}{2} \lambda^{2} \int_{0}^{l}\left(\theta^{\prime \prime}(\gamma(x)) \delta \gamma^{2}+w^{\prime \prime}(\beta-\bar{\gamma}) \delta \bar{\gamma}^{2}\right) d x+o\left(\lambda^{2}\right)
\end{aligned}
$$

the first variation be nonnegative for all perturbations $\delta \gamma$ and for all positive values of the smallness parameter $\lambda$. By (2-11) and (2-16), this is true if and only if $(\beta, \gamma)$ is an equilibrium configuration. Therefore, all minimizers for $E^{\mathrm{eq}}(\beta, \cdot)$ are equilibrium configurations.

A second necessary condition is that the second variation be nonnegative for all $\delta \gamma$ for which the first variation is zero. In particular, it must be nonnegative for all $\delta \gamma$ with support in the inelastic zone $\mathscr{F}(\beta, \gamma)$. For this, it is necessary that

$$
\theta^{\prime \prime}(\gamma(x)) \geq 0, \quad \text { a.e. } x \in \mathscr{I}(\beta, \gamma)
$$

Indeed, if $\theta^{\prime \prime}(\gamma(x))$ is negative on a subset of $\mathscr{G}(\beta, \gamma)$ with nonnull measure, a negative second variation is obtained by concentrating $\delta \gamma$ on that set. The existence of perturbed configurations $(\beta, \gamma+\delta \gamma)$ with arbitrarily large negative energy characterizes a fractured configuration. Then, (2-21) is in fact a safety condition against fracture.

The nonnegativeness of the first variation joined with the stronger condition

$$
\theta^{\prime \prime}(\gamma(x))>0, \quad \forall x \in(0, l)
$$

is sufficient for a local minimum at $\gamma$ with respect to the $L^{\infty}$ norm:

$$
\|\delta \gamma\|=\sup _{x \in(0, l)}|\delta \gamma(x)|
$$

Indeed, since $\theta$ is $C^{2}$ and $\gamma$ is continuous in the closed set $[0, l]$, the map $\theta^{\prime \prime}(\gamma(\cdot))$ is uniformly continuous. Then for all $\varepsilon>0$ there is a $\delta>0$ such that

$$
|a|<\delta \quad \Longrightarrow \quad\left|\theta^{\prime \prime}(\gamma(x)+a)-\theta^{\prime \prime}(\gamma(x))\right|<\varepsilon, \quad \forall x \in(0, l) .
$$

Consider the finite expansion of $E^{\mathrm{eq}}(\beta, \cdot)$ with Lagrange remainder

$$
\begin{aligned}
& E^{\mathrm{eq}}(\beta, \gamma+\delta \gamma) \\
& \quad=E^{\mathrm{eq}}(\beta, \gamma)+\delta E^{\mathrm{eq}}(\beta, \gamma, \delta \gamma)+\frac{1}{2} \int_{0}^{l}\left(\theta^{\prime \prime}(\gamma(x)+\xi(x) \delta \gamma(x)) \delta \gamma^{2}(x)+w^{\prime \prime}(\beta-\bar{\gamma}-\bar{\xi} \delta \bar{\gamma}) \delta \bar{\gamma}^{2}\right) d x,
\end{aligned}
$$

with $\bar{\xi}$ and all $\xi(x)$ in $(0,1)$. For every perturbation $\delta \gamma$ with $\|\delta \gamma\|<\delta$, from the nonnegativeness of the first variation and the positiveness of $w^{\prime \prime}$ we have

$$
E^{\mathrm{eq}}(\beta, \gamma+\delta \gamma)-E^{\mathrm{eq}}(\beta, \gamma) \geq \frac{1}{2} \int_{0}^{l}\left(\theta^{\prime \prime}(\gamma(x))-\varepsilon\right) \delta \gamma^{2}(x) d x
$$


If (2-22) holds, the map $\theta^{\prime \prime}(\gamma(\cdot))$ is positive and continuous, and therefore it has a positive minimum $c$ in $[0, l]$. It is then sufficient to take $\varepsilon<c$ to have a nonnegative right-hand side. Because this holds for all $\delta \gamma$ with $\|\delta \gamma\|<\delta, \gamma$ is a local minimizer with respect to the norm (2-23).

2.4. Quasistatic evolutions. A load process is a continuous function $t \mapsto \beta_{t}$. A quasistatic evolution from $\gamma_{0}$ associated with $t \mapsto \beta_{t}$ is a family $t \mapsto \gamma_{t}$ of inelastic deformations such that

(i) $\gamma_{t}=\gamma_{0}$ at the initial time $t=t_{0}$,

(ii) for all $t$ the function $x \mapsto \gamma_{t}(x)$ is continuous, for all $x$ the function $t \mapsto \gamma_{t}$ is nondecreasing, and the family $t \mapsto \gamma_{t}$ is continuous in $L^{\infty}(0, l)$, and

(iii) for all $t \geq t_{0}, \gamma_{t}$ is a local minimizer for $E^{\mathrm{eq}}\left(\beta_{t}, \cdot\right)$.

In (ii), the requirement of $\gamma_{t}$ nondecreasing is imposed by the dissipation inequality (2-10), and continuity in $L^{\infty}(0, l)$ means that for every $t \geq t_{0}$ and for every $\delta>0$ there is a $\tau_{o}>0$ such that

$$
\left\|\gamma_{t+\tau}-\gamma_{t}\right\|<\delta, \quad \forall \tau \in\left(0, \tau_{o}\right),
$$

where $\|\cdot\|$ is the $L^{\infty}$ norm (2-23). By consequence, in the time interval $\left(0, \tau_{o}\right)$ the evolution $\tau \mapsto \gamma_{t+\tau}$ takes place inside the ball $\mathscr{B}\left(\gamma_{t}, \delta\right)$ of $L^{\infty}(0, l)$ of radius $\delta$ centered at $\gamma_{t}$.

Assume that $\gamma_{t}(\cdot)$ is a deformation with constant value $\bar{\gamma}_{t}$, and that $\theta^{\prime \prime}\left(\bar{\gamma}_{t}\right)$ is positive. Then it is possible to choose $\delta$ such that $\theta^{\prime \prime}(\gamma(x))>0$ for all $\gamma$ in $\mathscr{B}\left(\bar{\gamma}_{t}, \delta\right)$, so that the restriction of the energy to $\mathscr{B}\left(\bar{\gamma}_{t}, \delta\right)$ is strictly convex for all $\tau<\tau_{o}$. Therefore, by Jensen's inequality,

$$
E^{\mathrm{eq}}\left(\beta_{t+\tau}, \gamma_{t+\tau}\right) \geq E^{\mathrm{eq}}\left(\beta_{t+\tau}, \bar{\gamma}_{t+\tau}\right), \quad \forall \tau \in\left(0, \tau_{o}\right),
$$

where $\bar{\gamma}_{t+\tau}$ is the homogeneous deformation defined as in (2-4), and equality holds only if $\gamma_{t+\tau}=\bar{\gamma}_{t+\tau}$. That is, the unique minimizer of the energy at time $t+\tau$ is a homogeneous deformation. Consequently, for every quasistatic evolution from a homogeneous deformation $\bar{\gamma}_{t}$ with $\theta^{\prime \prime}\left(\bar{\gamma}_{t}\right)>0$ there is a time interval $\left(0, \tau_{o}\right)$ in which $\gamma_{t+\tau}$ is a homogeneous deformation.

From here onwards we consider homogeneous deformation processes $t \mapsto \gamma_{t}$, and we identify the functions $\gamma_{t}(\cdot)$ and $\dot{\gamma}_{t}(\cdot)$ with their constant values, which we denote by $\gamma_{t}$ and $\dot{\gamma}_{t}$. For $\theta^{\prime \prime}\left(\gamma_{t}\right)>0$ and for $\delta$ and $\tau_{o}$ as above, the determination of a quasistatic evolution from $\left(\beta_{t}, \gamma_{t}\right)$ reduces to the following incremental minimization problem: for a given $\tau \leq \tau_{o}$, find the homogeneous deformation $\gamma_{t+\tau}$ which minimizes the energy $E^{\mathrm{eq}}\left(\beta_{t+\tau}, \cdot\right)$ in $\mathscr{B}\left(\gamma_{t}, \delta\right)$.

An approximate solution is obtained by replacing $\beta_{t+\tau}$ and $\gamma_{t+\tau}$ by the piecewise linear approximations

$$
\beta_{t+\tau} \approx \beta_{t}+\tau \dot{\beta}_{t}, \quad \gamma_{t+\tau} \approx \gamma_{t}+\tau \dot{\gamma}_{t},
$$

and then determining $\dot{\gamma}_{t}$ as the minimizer for the first-order approximation

$$
E_{t+\tau}(\dot{\gamma}) \approx E_{t}+\tau \dot{E}_{t}(\dot{\gamma})
$$

of the energy $E^{\mathrm{eq}}\left(\beta_{t+\tau}, \gamma_{t+\tau}\right)$, where

$$
E_{t}=E^{\mathrm{eq}}\left(\beta_{t}, \gamma_{t}\right)=l\left(w\left(\epsilon_{t}\right)+\theta\left(\gamma_{t}\right)\right), \quad \dot{E}_{t}(\dot{\gamma})=l\left(\sigma_{t}\left(\dot{\beta}_{t}-\dot{\gamma}\right)+\theta^{\prime}\left(\gamma_{t}\right) \dot{\gamma}\right),
$$


with $\sigma_{t}=w^{\prime}\left(\epsilon_{t}\right)$ and $\epsilon_{t}=\beta_{t}-\gamma_{t}$. This minimization determines the direction of steepest descent for the energy. Because $E_{t}$ and $\dot{\beta}_{t}$ are known, the problem reduces to the minimization of the linear function

$$
I(\dot{\gamma})=\left(\theta^{\prime}\left(\gamma_{t}\right)-\sigma_{t}\right) \dot{\gamma}
$$

under the condition $\dot{\gamma} \geq 0$. In the elastic regime, in which $\left(\theta^{\prime}\left(\gamma_{t}\right)-\sigma_{t}\right)$ is positive, the minimum is zero and is attained at $\dot{\gamma}=0$. In the inelastic regime, $I(\dot{\gamma})$ is identically zero because $\left(\theta^{\prime}\left(\gamma_{t}\right)-\sigma_{t}\right)$ is zero. That is, the direction of steepest descent is undetermined.

The determination of a quasistatic evolution in the inelastic regime then requires a second-order approximation of the energy

$$
E_{t+\tau}(\dot{\gamma}) \approx E_{t}+\tau \dot{E}_{t}(\dot{\gamma})+\frac{1}{2} \tau^{2} \ddot{E}_{t}(\dot{\gamma})
$$

where

$$
\ddot{E}_{t}(\dot{\gamma})=l\left(w^{\prime \prime}\left(\epsilon_{t}\right)\left(\dot{\beta}_{t}-\dot{\gamma}\right)^{2}+\theta^{\prime \prime}\left(\gamma_{t}\right) \dot{\gamma}^{2}\right)
$$

After elimination of the terms independent of $\dot{\gamma}$, the problem reduces to the minimization of the quadratic function

$$
J(\dot{\gamma})=\left(\theta^{\prime}\left(\gamma_{t}\right)-\sigma_{t}-\tau w^{\prime \prime}\left(\epsilon_{t}\right) \dot{\beta}_{t}\right) \dot{\gamma}+\frac{1}{2} \tau\left(\theta^{\prime \prime}\left(\gamma_{t}\right)+w^{\prime \prime}\left(\epsilon_{t}\right)\right) \dot{\gamma}^{2},
$$

under the condition $\dot{\gamma} \geq 0$. The sum $\left(\theta^{\prime \prime}\left(\gamma_{t}\right)+w^{\prime \prime}\left(\epsilon_{t}\right)\right)$ is positive by the strict convexity of $w$ and the necessary condition (2-21) on $\theta^{\prime \prime}(\gamma)$. Then, $J$ is strictly convex. If the bar is in the elastic regime, $\theta^{\prime}\left(\gamma_{t}\right)-\sigma_{t}>0$, the coefficient of $\dot{\gamma}$ is positive for sufficiently small $\tau$. Then the global minimum is zero, and is achieved at $\dot{\gamma}=0$. In this case, the elastic incremental response

$$
\dot{\sigma}_{t}=w^{\prime \prime}\left(\varepsilon_{t}\right) \dot{\beta}_{t}
$$

follows from the incremental force-elongation relation

$$
\dot{\sigma}_{t}=w^{\prime \prime}\left(\varepsilon_{t}\right)\left(\dot{\beta}_{t}-\overline{\dot{\gamma}}_{t}\right)
$$

obtained by time differentiation of (2-17). This confirms the result of the first-order minimization, that there is no increase of inelastic deformation in the elastic regime.

In the inelastic regime, $\theta^{\prime}\left(\gamma_{t}\right)=\sigma_{t}$, the minimum is still achieved at $\dot{\gamma}=0$ at unloading, $\dot{\beta}_{t} \leq 0$. Then, there is no increase of inelastic deformation at unloading. At loading, $\dot{\beta}_{t}>0$, the minimum of $J$ is achieved at

$$
\dot{\gamma}_{t}=\frac{w^{\prime \prime}\left(\epsilon_{t}\right)}{\theta^{\prime \prime}\left(\gamma_{t}\right)+w^{\prime \prime}\left(\epsilon_{t}\right)} \dot{\beta}_{t},
$$

and, by (2-32), the inelastic response is

$$
\dot{\sigma}_{t}=\frac{\theta^{\prime \prime}\left(\gamma_{t}\right) w^{\prime \prime}\left(\epsilon_{t}\right)}{\theta^{\prime \prime}\left(\gamma_{t}\right)+w^{\prime \prime}\left(\epsilon_{t}\right)} \dot{\beta}_{t} .
$$

The different response at loading and unloading in the inelastic regime is a peculiar property of plastic behavior. At loading, from (2-33) and (2-34) it follows that

$$
\dot{\sigma}_{t}=\theta^{\prime \prime}\left(\gamma_{t}\right) \dot{\gamma}_{t}
$$


whence

$$
\sigma_{t+\tau} \approx \sigma_{t}+\tau \dot{\sigma}_{t}=\theta^{\prime}\left(\gamma_{t}\right)+\tau \theta^{\prime \prime}\left(\gamma_{t}\right) \dot{\gamma}_{t} \approx \theta^{\prime}\left(\gamma_{t+\tau}\right)
$$

Equation (2-35) is the hardening rule, which in classical plasticity specifies $\dot{\sigma}$ as a function of the current value of $\gamma$. Equation (2-36) states that, within the approximation (2-24), at time $t+\tau$ the yield condition holds as an equality. That is, the inelastic regime is preserved. This is the consistency condition of classical plasticity. At unloading, the response (2-31) expresses the property of elastic unloading. Classically, the hardening rule, consistency, and elastic unloading are assumptions originating from experimental observation. Here, they come as necessary conditions for a minimum in the second-order energy minimization.

Equation (2-34) provides the slope $d \sigma_{t} / d \beta_{t}=\dot{\sigma}_{t} / \dot{\beta}_{t}$ of the force-elongation response curve at loading. For $\theta^{\prime \prime}\left(\gamma_{t}\right)>0$ this slope is positive but smaller than the slope $w^{\prime \prime}\left(\epsilon_{t}\right)$ at unloading. A positive slope at loading characterizes a work-hardening response.

For $\theta^{\prime \prime}\left(\gamma_{t}\right)=0$, the restriction to homogeneous deformations is no longer possible. In the inelastic regime, $\sigma_{t}=\theta^{\prime}\left(\gamma_{t}\right)$, the function $J(\dot{\gamma})$ reduces to

$$
J(\dot{\gamma})=-\tau w^{\prime \prime}\left(\epsilon_{t}\right) \dot{\beta}_{t} \overline{\dot{\gamma}}+\frac{1}{2} \tau w^{\prime \prime}\left(\epsilon_{t}\right) \overline{\dot{\gamma}}^{2} .
$$

The minimum is achieved at $\overline{\dot{\gamma}}_{t}=\dot{\beta}_{t}$, which corresponds to $\dot{\sigma}_{t}=0$, that is, to perfectly plastic response. In this case, the incremental minimization specifies the average $\overline{\dot{\gamma}}_{t}$, but the punctual values $\dot{\gamma}_{t}(x)$ are not determined. Thus, in this case the evolution from a homogeneous configuration need not be homogeneous.

The case of $\theta^{\prime \prime}\left(\gamma_{t}\right)$ negative, corresponding to a negative slope, that is, to a strain-softening response, is excluded by condition (2-21). Multiplication of (2-35) by $\dot{\gamma}_{t}$ shows that Drucker's postulate (1-5) is obeyed only if $\theta^{\prime \prime}\left(\gamma_{t}\right)$ is nonnegative. This shows that the local model provides a valid response only for materials which obey Drucker's postulate.

The indeterminacy of the minimizers in a perfectly plastic response and the impossibility of reproducing the softening response are two major drawbacks of the local model.

2.5. Evolution from the natural configuration. Let us determine the quasistatic evolution for a load process $t \mapsto \beta_{t}$, with $\dot{\beta}_{t}>0$ for all $t$, from the natural configuration $\left(\beta_{0}, \gamma_{0}\right)=(0,0)$. At $t=0$ we have

$$
\sigma_{0}=0, \quad \theta^{\prime}\left(\gamma_{0}\right)=\theta^{\prime}(0)>0,
$$

so that inequality (2-20) is strict and $\dot{\gamma}_{0}$ is zero. By continuity, inequality (2-20) remains strict, and therefore $\dot{\gamma}_{t}$ remains equal to zero, over a time interval $\left(0, t_{c}\right)$. Because $\gamma_{0}=0, \gamma_{t}$ is zero in this interval. Then an elastic regime takes place, with

$$
\epsilon_{t}=\beta_{t}, \quad \sigma_{t}=w^{\prime}\left(\beta_{t}\right) .
$$

This regime ends when, at some $t_{c}>0, \beta_{t}$ reaches the value

$$
\beta_{c}=\left(w^{\prime}\right)^{-1}\left(\theta^{\prime}(0)\right)
$$

at which, since $\sigma_{t_{c}}=w^{\prime}\left(\beta_{c}\right)=\theta^{\prime}(0)$, inequality (2-20) becomes an equality. At $t=t_{c}$, all points of the bar switch from the elastic to the inelastic zone. 
The response at $t=t_{c}$ is work-hardening if $\theta^{\prime \prime}(0)>0$ and perfectly plastic if $\theta^{\prime \prime}(0)=0$. In the work-hardening regime, by (2-33), the evolution $t \mapsto \gamma_{t}$ is determined by the solution of the differential equation

$$
\dot{\gamma}_{t}=\frac{w^{\prime \prime}\left(\beta_{t}-\gamma_{t}\right)}{\theta^{\prime \prime}\left(\gamma_{t}\right)+w^{\prime \prime}\left(\beta_{t}-\gamma_{t}\right)} \dot{\beta}_{t}, \quad t \geq t_{c},
$$

under the initial condition $\gamma_{t_{c}}=0$. A work-hardening regime persists as long as $\dot{\beta}_{t}$ and $\theta^{\prime \prime}\left(\gamma_{t}\right)$ remain positive. For $\dot{\beta}_{t} \leq 0$ elastic unloading takes place, and for $\theta^{\prime \prime}\left(\gamma_{t}\right)=0$ the response becomes perfectly plastic. Fracture occurs as soon as $\gamma_{t}$ becomes larger than

$$
\gamma_{r} \doteq \inf \left\{\gamma \geq 0 \mid \theta^{\prime \prime}(\gamma)<0\right\}
$$

Indeed, for $\gamma>\gamma_{r}$ there are perturbed configurations with arbitrarily large negative energy. Because the energy suddenly drops from a finite value to $-\infty$, the fracture has a catastrophic character. This fracture mode is called brittle fracture. If $\theta^{\prime \prime}(0)<0$, fracture occurs without any intermediate inelastic regime. This is the case of totally brittle fracture.

In conclusion, in the inelastic regime at loading the response at $\left(\beta_{t}, \gamma_{t}\right)$ is work-hardening if $\theta^{\prime \prime}\left(\gamma_{t}\right)$ is positive, and perfectly plastic if $\theta^{\prime \prime}\left(\gamma_{t}\right)=0$. For $\theta^{\prime \prime}\left(\gamma_{t}\right)>0$, a quasistatic evolution from a homogeneous deformation is made of homogeneous deformations. The fracture is always catastrophic, and occurs as soon as $\gamma_{t}$ becomes larger than $\gamma_{r}$.

\section{The nonlocal model}

3.1. Basic assumptions. The local model provides only a rough description of the bar's behavior. In particular, it does not describe ductile fracture and strain-softening response. These phenomena are captured by adding to the energy (2-5) a nonlocal term proportional to the square of the derivative of $\gamma$ :

$$
E(\epsilon, \gamma)=\int_{0}^{l}\left(w(\epsilon(x))+\theta(\gamma(x))+\frac{1}{2} \alpha \gamma^{\prime 2}(x)\right) d x
$$

with $\alpha$ a positive constant. The new term requires a stronger regularity of $\gamma$. We assume that $\gamma$ is $C^{1}$ on $[0, l]$ and piecewise $C^{2}$, that is, that $\gamma^{\prime \prime}$ is continuous except at a finite number of jump points $x_{i}$, at which the left limit $\gamma^{\prime \prime}\left(x_{i}-\right)$ and the right limit $\gamma^{\prime \prime}\left(x_{i}+\right)$ exist. The difference

$$
\left[\left[\gamma^{\prime \prime}\right]\right]\left(x_{i}\right) \doteq \gamma^{\prime \prime}\left(x_{i}+\right)-\gamma^{\prime \prime}\left(x_{i}-\right)
$$

is the jump of $\gamma^{\prime \prime}$ at $x$.

Like in the local model, the part of the energy depending on the inelastic deformation is supposed to be dissipative. That is, the cohesive power

$$
\theta^{\prime}\left(\gamma_{t}(x)\right) \dot{\gamma}_{t}(x)+\alpha \gamma_{t}^{\prime}(x) \dot{\gamma}_{t}^{\prime}(x)
$$

is assumed to be nonnegative in every deformation process $t \mapsto \gamma_{t}$. For this, it is necessary that the product $\theta^{\prime}\left(\gamma_{t}(x)\right) \dot{\gamma}_{t}(x)$ be nonnegative at all $x$. Indeed, if it is negative at some $x_{o}$, since $\dot{\gamma}_{t}^{\prime}\left(x_{o}\right)$ may have opposite signs in different deformation processes from $\gamma_{t}\left(x_{o}\right)$, for at least one choice the power (3-2) is negative. 
By the assumed strict monotonicity of $\theta^{\prime}$, the dissipation inequality $\dot{\gamma}_{t}(x) \geq 0$ is necessary for dissipativity. In the following subsection we show that, if the yield limit is nonnegative, this condition is sufficient to guarantee the global dissipativity, that is, the nonnegativeness of the integral of (3-2) over $(0, l)$, in every equilibrium process.

3.2. Equilibrium. In the nonlocal model, an equilibrium configuration is a configuration $(\epsilon, \gamma)$ at which the first variation

$$
\delta E(\epsilon, \gamma, \delta \epsilon, \delta \gamma)=\int_{0}^{l}\left(w^{\prime}(\epsilon(x)) \delta \epsilon(x)+\theta^{\prime}(\gamma(x)) \delta \gamma(x)+\alpha \gamma^{\prime}(x) \delta \gamma^{\prime}(x)\right) d x
$$

is nonnegative for all $C^{1}([0, l])$ and piecewise $C^{2}$ perturbations $(\delta \epsilon, \delta \gamma)$, which satisfy the dissipation inequality (2-11) and the boundary condition (2-13). Proceeding as in Section 2.2, using perturbations with $\delta \gamma=0$ we find that $\sigma$ and $\epsilon$ must be constant over the bar. Then setting $w^{\prime}(\epsilon)=\sigma$ and $\delta \epsilon=-\delta \bar{\gamma}$ and integrating by parts, we get

$$
\delta E(\epsilon, \gamma,-\delta \bar{\gamma}, \delta \gamma)=\int_{0}^{l}\left(\theta^{\prime}(\gamma(x))-\sigma-\alpha \gamma^{\prime \prime}(x)\right) \delta \gamma(x) d x+\alpha\left[\gamma^{\prime}(x) \delta \gamma(x)\right]_{0}^{l} .
$$

The nonnegativeness of the first variation for all nonnegative $\delta \gamma$ requires

$$
\sigma \leq \theta^{\prime}(\gamma(x))-\alpha \gamma^{\prime \prime}(x),
$$

in $(0, l)$, and

$$
\gamma^{\prime}(l) \delta \gamma(l) \geq 0, \quad \gamma^{\prime}(0) \delta \gamma(0) \leq 0,
$$

at the boundary. Inequality (3-3) is the nonlocal version of the yield condition (2-20). The difference with the local model is that, while the yield limit $\theta^{\prime}(\gamma(x))$ of the local model depends only on the value of $\gamma$ at $x$, the additional term $\alpha \gamma^{\prime \prime}(x)$ introduces a dependence on the values taken by $\gamma$ at the neighboring points.

There are two ways of satisfying the boundary conditions (3-4). If $\delta \gamma$ is allowed to take arbitrary positive values at the boundary, these conditions reduce to $\gamma^{\prime}(l) \geq 0$ and $\gamma^{\prime}(0) \leq 0$, respectively. The alternative is to require that

$$
\gamma(l)=\gamma(0)=0,
$$

and, therefore, to take perturbations $\delta \gamma$ such that

$$
\delta \gamma(l)=\delta \gamma(0)=0 .
$$

We choose this second possibility, which better describes the standard experimental conditions. Indeed, while this choice keeps the inelastic deformations away from the boundary, the other choice favors the concentration of the inelastic deformation at the boundary. For a similar effect in different models, see [Yalcinkaya et al. 2011, Figures 9 and 10], [Benallal and Marigo 2007, Figure 3a], and the discussion in [Jirásek and Rolshoven 2009b]. In laboratory tests this effect is carefully avoided, either by reinforcing the specimen's end sections, or by weakening the central part of the bar, for example, with the creation of a notch.

Thus, our choice allows for a comparison, at least qualitative, with the standard experimental curves. The alternative possibility of imposing a null derivative $\gamma^{\prime}$ was chosen in [Benallal and Marigo 2007; 
Pham and Marigo 2010b; Pham et al. 2011]. This choice has the advantage of allowing for homogeneous inelastic equilibrium configurations, and this greatly facilitates the study of the inelastic regime. Unfortunately, due to the devices used to fix the bar's ends in real experiments, this circumstance is hardly reproducible in practice.

In conclusion, for the nonlocal model an equilibrium configuration is a pair $(\epsilon, \gamma)$, with $\epsilon$ a constant and $\gamma$ a $C^{1}([0, l])$ and piecewise $C^{2}$ function, which satisfies inequality (3-3) at the interior points and conditions (3-5) at the boundary.

Assume that the dissipation inequality (2-10) holds, and that the yield limit $\theta^{\prime}(\gamma)-\alpha \gamma^{\prime \prime}$ is nonnegative. After integrating over $(0, l)$, an integration by parts yields

$$
0 \leq \int_{0}^{l}\left(\theta^{\prime}(\gamma(x))-\alpha \gamma^{\prime \prime}(x)\right) \dot{\gamma}(x) d x=\int_{0}^{l}\left(\theta^{\prime}(\gamma(x)) \dot{\gamma}(x)+\alpha \gamma^{\prime}(x) \dot{\gamma}^{\prime}(x)\right) d x-\alpha\left[\gamma^{\prime}(x) \dot{\gamma}(x)\right]_{0}^{l} .
$$

On the right side the integral is the total cohesive power. Moreover, by (3-4), the remaining term is nonpositive in any equilibrium process. This proves that, if the yield limit is nonnegative, the dissipation inequality (2-10) guarantees the global dissipativity in every equilibrium process.

An equilibrium configuration $(\epsilon, \gamma)$ is equilibrated with the load $\beta$ given by (2-3). Alternatively, an equilibrium configuration can be defined as a pair $(\beta, \gamma)$, and the energy can be redefined by

$$
E^{\mathrm{eq}}(\beta, \gamma)=l w(\beta-\bar{\gamma})+\int_{0}^{l} \theta(\gamma(x)) d x+\frac{1}{2} \alpha \int_{0}^{l} \gamma^{\prime 2}(x) d x .
$$

For an equilibrium configuration $(\beta, \gamma)$ defined in this way, the elastic and inelastic zones are

$$
\mathscr{E}(\beta, \gamma)=\left\{x \in(0, l) \mid w^{\prime}(\beta-\bar{\gamma})<\theta^{\prime}(\gamma(x))-\alpha \gamma^{\prime \prime}(x)\right\}, \quad \mathscr{J}(\beta, \gamma)=(0, l) \backslash \mathscr{E}(\beta, \gamma),
$$

respectively. As in the local model, we say that the bar is in the elastic regime if $\mathscr{E}(\beta, \gamma)=(0, l)$, and that it is in the inelastic regime otherwise.

3.3. Energy minimizers. To determine the inelastic deformations $\gamma$ which minimize the energy $E^{\mathrm{eq}}(\beta, \cdot)$, consider the expansion

$$
\begin{aligned}
E^{\mathrm{eq}}(\beta, \gamma+\delta \gamma)-E^{\mathrm{eq}}(\beta, \gamma) & =\int_{0}^{l}\left(\theta^{\prime}(\gamma(x)) \delta \gamma(x)-w^{\prime}(\epsilon) \delta \bar{\gamma}+\alpha \gamma^{\prime}(x) \delta \gamma^{\prime}(x)\right) d x \\
& +\frac{1}{2} \int_{0}^{l}\left(\theta^{\prime \prime}(\gamma(x)) \delta \gamma^{2}(x)+w^{\prime \prime}(\epsilon) \delta \bar{\gamma}^{2}+\alpha \delta \gamma^{\prime 2}(x)\right) d x+o\left(\|\delta \gamma\|^{2}\right),
\end{aligned}
$$

with $\epsilon=\beta-\bar{\gamma}$, and with perturbations $\delta \gamma$ nonnegative and satisfying the boundary conditions (3-6). A necessary condition for a minimum is the nonnegativeness of the first variation. Because this requirement characterizes an equilibrium configuration, all minimizers are equilibrium configurations.

Another necessary condition is that the second variation be nonnegative for all $\delta \gamma$ for which the first variation is zero, and in particular, for all $\delta \gamma$ with support in the inelastic zone $\mathscr{f}(\beta, \gamma)$. If $\left(a, a+l_{j}\right)$ is an interval in the inelastic zone, the second variation is nonnegative only if the smallest eigenvalue of the eigenvalue problem

$$
\int_{a}^{a+l_{J}}\left(\theta^{\prime \prime}(\gamma(x)) \delta \gamma^{2}(x)+\frac{l}{l_{J}} w^{\prime \prime}(\epsilon) \delta \bar{\gamma}^{2}+\alpha \delta \gamma^{\prime 2}(x)\right) d x=\alpha \rho \int_{a}^{a+l_{J}} \delta \gamma^{2}(x) d x,
$$


is nonnegative for all perturbations $\delta \gamma$ with support in this interval. This problem can be identified with problem (A.15), with $H$ as in (A.9) due to the presence of the constraint $\delta \gamma(x) \geq 0$, and with

$$
a=0, \quad l_{J}=L, \quad \theta^{\prime \prime}(\gamma(x))=\alpha h(x), \quad \delta \gamma(x)=y(x), \quad \delta \bar{\gamma}=\frac{l_{J}}{l} \bar{y} .
$$

The last equality follows from the definitions

$$
\bar{y}=\frac{1}{l_{J}} \int_{a}^{a+l_{\jmath}} y(x) d x, \quad \delta \bar{\gamma}=\frac{1}{l} \int_{0}^{l} \delta \gamma(x) d x=\frac{1}{l} \int_{0}^{l_{j}} \delta \gamma(x) d x,
$$

which hold for $\delta \gamma(x)=y(x)$ with support in $\left(0, l_{J}\right)$. Then, from the identification

$$
\frac{l}{l_{J}} w^{\prime \prime}(\epsilon) \delta \bar{\gamma}^{2}=\alpha \omega \bar{y}^{2},
$$

comes the relation

$$
\omega=\frac{l_{J}}{l} \frac{w^{\prime \prime}(\epsilon)}{\alpha} .
$$

The smallest eigenvalue $\alpha \rho_{o}$ has the upper bound (A.17). It provides the necessary condition for nonnegativeness:

$$
\alpha \lambda_{o_{J}}^{2}+\theta_{o_{J}}^{\prime \prime} \geq 0
$$

where $\lambda_{o_{J}}$ is a solution of (A.14), now rewritten in the form

$$
\lambda_{o_{J}}^{2}=\frac{l_{J}}{l} \frac{w^{\prime \prime}(\epsilon)}{\alpha} \psi_{o}\left(\lambda_{o_{J}} l_{J}\right)
$$

with $\psi_{o}$ as in (A.13). Moreover, $\theta_{o j}^{\prime \prime}$ is defined as in (A.16),

$$
\theta_{o_{J}}^{\prime \prime}=\frac{\int_{a}^{a+l_{J}} \theta^{\prime \prime}(\gamma(x)) y_{o_{J}}^{2}(x) d x}{\int_{a}^{a+l_{J}} y_{o_{J}}^{2}(x) d x},
$$

with $y_{o J}$ given by (A.8) if $\omega L^{2}=l_{J}^{3} w^{\prime \prime}(\epsilon) / \alpha l \leq 4 \pi^{2}$, and given by (A.12) if $l_{J}^{3} w^{\prime \prime}(\epsilon) / \alpha l>4 \pi^{2}$. Because $\lambda_{o J}$ is a decreasing function of $l_{J}$, inequality (3-10) must be satisfied for the largest interval contained in the inelastic zone.

To find a sufficient condition for a local minimum of $E^{\mathrm{eq}}(\beta, \gamma)$, write the finite expansion with a Lagrange remainder:

$$
\begin{aligned}
E^{\mathrm{eq}}(\beta, \gamma+\delta \gamma) & =E^{\mathrm{eq}}(\beta, \gamma)+\int_{0}^{l}\left(\theta^{\prime}(\gamma(x)) \delta \gamma(x)-w^{\prime}(\epsilon) \delta \bar{\gamma}+\alpha \gamma^{\prime}(x) \delta \gamma^{\prime}(x)\right) d x \\
& +\frac{1}{2} \int_{0}^{l}\left(\theta^{\prime \prime}(\gamma(x)+\xi(x) \delta \gamma(x)) \delta \gamma^{2}(x)+w^{\prime \prime}(\epsilon-\bar{\xi} \delta \bar{\gamma}) \delta \bar{\gamma}^{2}+\alpha \delta \gamma^{\prime 2}(x)\right) d x,
\end{aligned}
$$

with $0 \leq \xi(x) \leq 1$ for all $x$ and $0 \leq \bar{\xi} \leq 1$. The first integral, which is the first variation, is nonnegative. We wish to find conditions for which the second integral is nonnegative as well. 
By the continuity of $\theta^{\prime \prime}$ and $w^{\prime \prime}$, for every $\varepsilon>0$ there is a positive $\delta$ such that

$$
\sup _{x \in(0, l)}\left|\theta^{\prime \prime}(\gamma(x)+\xi(x) \delta \gamma(x))-\theta^{\prime \prime}(\gamma(x))\right|<\varepsilon, \quad\left|w^{\prime \prime}(\epsilon-\bar{\xi} \delta \bar{\gamma})-w^{\prime \prime}(\epsilon)\right|<\varepsilon,
$$

for all $\delta \gamma$ with $\|\delta \gamma\|<\delta$. For all such $\delta \gamma$, the second integral is greater than or equal to

$$
\int_{0}^{l}\left(\theta^{\prime \prime}(\gamma(x)) \delta \gamma^{2}(x)+w^{\prime \prime}(\epsilon) \delta \bar{\gamma}^{2}+\alpha \delta \gamma^{\prime 2}(x)\right) d x-\varepsilon \int_{0}^{l}\left(\delta \gamma^{2}(x)+\delta \bar{\gamma}^{2}\right) d x .
$$

The first integral is the same as in (A.15), with

$$
l=L, \quad \delta \gamma=y, \quad w^{\prime \prime}(\epsilon)=\alpha \omega, \quad \theta^{\prime \prime}(\gamma(x))=\alpha h(x) .
$$

Therefore,

$$
E^{\mathrm{eq}}(\beta, \gamma+\delta \gamma) \geq E^{\mathrm{eq}}(\beta, \gamma)+\left(\alpha \rho_{o}-2 \varepsilon\right) \int_{0}^{l} \delta \gamma^{2}(x) d x
$$

By the arbitrariness of $\varepsilon, \gamma$ is a minimizer if the smallest eigenvalue $\rho_{o}$ is strictly positive. By (A.19), a sufficient condition for a minimum is

$$
\alpha \lambda_{o}^{2}+\theta_{\min }^{\prime \prime}>0
$$

with $\lambda_{o}$ the solution of (A.14) for the given $\omega$, and with $\theta_{\min }^{\prime \prime}$ the smallest value of $\theta^{\prime \prime}(\gamma(x))$ in $(0, l)$.

Conditions (3-10) and (3-13) improve the corresponding conditions (2-21) and (2-22) of the local model. In particular, they show that moderate negative values of $\theta^{\prime \prime}(\gamma(x))$ are allowed in an energy minimizer. As remarked in the Appendix, the two conditions can be far away from each other. In the special case of $l_{J}=l$ and constant $\theta^{\prime \prime}(\gamma(x))$ they coincide, save for the fact that the second inequality is strict.

3.4. Quasistatic evolutions. For a given load process $t \mapsto \beta_{t}$, a quasistatic evolution from a given deformation $\gamma_{0}$ at $t=t_{0}$ is a family $t \mapsto \gamma_{t}$ of inelastic deformations such that

(i) $\gamma_{t_{0}}$ is equal to $\gamma_{0}$,

(ii) for all $t \geq t_{0}$, the function $\gamma_{t}(\cdot)$ is $C^{1}$ and piecewise $C^{2}$ in $[0, l]$,

(iii) for all $t \geq t_{0}, \gamma_{t}$ is a local minimizer for $E^{\mathrm{eq}}\left(\beta_{t}, \cdot\right)$.

The regularity of the time derivative $\dot{\gamma}_{t}$ is determined by Hadamard's kinematic compatibility condition:

$$
\llbracket \dot{\gamma}_{t}^{\prime} \rrbracket(x)=-\llbracket \gamma_{t}^{\prime \prime} \rrbracket(x) \frac{d x}{d t},
$$

where $d x / d t$ is the velocity of a moving jump of $\gamma_{t}^{\prime}$. This condition tells us that for a moving jump of $\gamma_{t}^{\prime \prime}$ there is a corresponding moving jump of $\dot{\gamma}_{t}^{\prime}$. Therefore, if $\gamma_{t}$ is $C^{1}$ and piecewise $C^{2}, \dot{\gamma}_{t}$ is only continuous, piecewise $C^{1}$, and piecewise $C^{2}$.

Like in the local model, item (iii) implies that all configurations $\left(\beta_{t}, \gamma_{t}\right)$ are equilibrium configurations. Note that, because of the boundary conditions (3-5), $\gamma_{t}$ is homogeneous only if $\gamma_{t}=0$. Therefore, while in the local model it is sometimes possible to restrict the analysis to quasistatic evolutions made of homogeneous deformations, here there are no homogeneous deformations besides $\gamma_{t}=0$. 
Repeating the procedure followed for the local model, fix a time step $\tau$ and consider the piecewise linear approximations (2-24) of $t \mapsto \beta_{t}$ and $t \mapsto \gamma_{t}$. For them, we first determine the direction $\dot{\gamma}_{t}$ of steepest descent, by minimizing the first-order approximation (2-25) of the energy. The problem reduces to the minimization of the linear functional

$$
I(\dot{\gamma})=\int_{0}^{l}\left(\theta^{\prime}\left(\gamma_{t}(x)\right) \dot{\gamma}(x)-\sigma_{t} \overline{\dot{\gamma}}+\alpha \gamma_{t}^{\prime}(x) \dot{\gamma}^{\prime}(x)\right) d x=\int_{0}^{l} f_{t}(x) \dot{\gamma}(x) d x,
$$

where $\sigma_{t}=w^{\prime}\left(\beta_{t}-\bar{\gamma}_{t}\right)$, and

$$
f_{t}(x) \doteq \theta^{\prime}\left(\gamma_{t}(x)\right)-\sigma_{t}-\alpha \gamma_{t}^{\prime \prime}(x)
$$

is the yield function. The domain of $I$ is the set of all $\dot{\gamma}$ which satisfy the dissipation inequality (2-10) and the boundary conditions

$$
\dot{\gamma}(0)=\dot{\gamma}(l)=0,
$$

which follow from (3-6). In the first variation

$$
\delta I\left(\dot{\gamma}_{t}, \delta \dot{\gamma}\right)=\int_{0}^{l} f_{t}(x) \delta \dot{\gamma}(x) d x,
$$

the perturbations $\delta \dot{\gamma}$ satisfy the boundary conditions

$$
\delta \dot{\gamma}(0)=\delta \dot{\gamma}(l)=0,
$$

and the inequality

$$
\dot{\gamma}_{t}(x)+\delta \dot{\gamma}(x) \geq 0, \quad \forall x \in(0, l) .
$$

This is because, in a quasistatic evolution, $\left(\dot{\gamma}_{t}+\delta \dot{\gamma}\right)$ is the derivative of $\gamma_{t}$ in some deformation process starting from $\left(\beta_{t}, \gamma_{t}\right)$, and therefore it obeys the boundary conditions (3-15) and the dissipation inequality $(2-10)$. The nonnegativeness of the first variation requires that

$$
f_{t}(x) \delta \dot{\gamma}(x) \geq 0, \quad \text { a.e. } x \in(0, l) .
$$

By (3-17), at points at which $\dot{\gamma}_{t}(x)=0$ the perturbation $\delta \dot{\gamma}(x)$ is arbitrary nonnegative, and therefore $f_{t}(x)$ must be nonnegative. At points at which $\dot{\gamma}_{t}(x)>0$ the perturbation may have any sign, and therefore $f_{t}(x)$ must be zero. Together with the dissipation inequality (2-10) and the yield condition (3-3), this leads to the Kuhn-Tucker conditions

$$
\dot{\gamma}_{t}(x) \geq 0, \quad f_{t}(x) \geq 0, \quad f_{t}(x) \dot{\gamma}_{t}(x)=0, \quad \text { a.e. } x \in(0, l),
$$

as necessary conditions for a minimum of $I$ at $\dot{\gamma}_{t}$. The last equality, called the complementarity condition, requires that $\dot{\gamma}_{t}(x)=0$ if $f_{t}(x)>0$, that is, at all points in the elastic zone $\mathscr{E}\left(\beta_{t}, \gamma_{t}\right)$.

For all $\dot{\gamma}_{t}$ which satisfy conditions (3-18), $I\left(\dot{\gamma}_{t}\right)$ is zero and $\dot{E}_{t}\left(\dot{\gamma}_{t}\right)$ is equal to $l \sigma_{t} \dot{\beta}_{t}$. Thus, like in the local model, the direction $\dot{\gamma}_{t}$ of steepest descent is not determined in the inelastic zone. For this, it is necessary to minimize the second-order approximation (2-28) of the energy. This reduces to the minimization of the functional

$$
J(\dot{\gamma})=\int_{0}^{l} f_{t}(x) \dot{\gamma}(x) d x+\frac{1}{2} \tau\left(\int_{0}^{l}\left(\theta^{\prime \prime}\left(\gamma_{t}(x)\right) \dot{\gamma}^{2}(x)+w^{\prime \prime}\left(\epsilon_{t}\right) \overline{\dot{\gamma}}^{2}+\alpha \dot{\gamma}^{\prime 2}(x)\right) d x-2 l w^{\prime \prime}\left(\epsilon_{t}\right) \dot{\beta_{t}} \overline{\dot{\gamma}}\right),
$$


with respect to all perturbations $\delta \dot{\gamma}$ which satisfy conditions (3-16) and (3-17). For the minimizers of $J$ we keep the same symbol $\dot{\gamma}_{t}$ used for the minimizers of $I$. While a minimizer for $I$ is a direction of steepest descent, a minimizer for $J$ will be called a continuation of the quasistatic evolution at $t$. The first variation of $J$ is

$$
\begin{aligned}
\delta J\left(\dot{\gamma}_{t}, \delta \dot{\gamma}\right) & =\int_{0}^{l} f_{t}(x) \delta \dot{\gamma}(x) d x+\tau \int_{0}^{l}\left(\theta^{\prime \prime}\left(\gamma_{t}(x)\right) \dot{\gamma}_{t}(x) \delta \dot{\gamma}(x)-\dot{\sigma}_{t} \delta \dot{\gamma}(x)+\alpha \dot{\gamma}_{t}^{\prime}(x) \delta \dot{\gamma}^{\prime}(x)\right) d x \\
& =\int_{0}^{l} f_{t}(x) \delta \dot{\gamma}(x) d x+\tau\left(\int_{0}^{l}\left(\theta^{\prime \prime}\left(\gamma_{t}(x)\right) \dot{\gamma}_{t}(x)-\dot{\sigma}_{t}-\alpha \dot{\gamma}_{t}^{\prime \prime}(x)\right) \delta \dot{\gamma}(x) d x-\alpha \sum_{i} \llbracket \dot{\gamma}_{t}^{\prime} \rrbracket\left(x_{i}\right) \delta \dot{\gamma}\left(x_{i}\right)\right) \\
& =\int_{0}^{l}\left(f_{t}(x)+\tau \dot{f}_{t}(x)\right) \delta \dot{\gamma}(x) d x-\tau \alpha \sum_{i} \llbracket \dot{\gamma}_{t}^{\prime} \rrbracket\left(x_{i}\right) \delta \dot{\gamma}\left(x_{i}\right) .
\end{aligned}
$$

The derivative $\dot{\gamma}_{t}^{\prime \prime}(x)$ which appears in $\dot{f}_{t}(x)$ is the regular part of the distributional derivative of $\dot{\gamma}_{t}^{\prime}$, while the singular part is given by the jumps $\left.\left[\dot{\gamma}_{t}^{\prime}\right]\right]\left(x_{i}\right)$ at the jump points $x_{i}$. The necessary conditions for a minimum at $\dot{\gamma}=\dot{\gamma}_{t}$ are

$$
\begin{aligned}
\left(f_{t}(x)+\tau \dot{f}_{t}(x)\right) \delta \dot{\gamma}(x) & \geq 0, & & \text { a.e. } x \in(0, l), \\
\llbracket\left[\dot{\gamma}_{t}^{\prime} \rrbracket(x) \delta \dot{\gamma}(x)\right. & \leq 0, & & \forall x \in(0, l) .
\end{aligned}
$$

The jump condition has been imposed at all $x$ and not only at the jump points, since $\llbracket \dot{\gamma}_{t}^{\prime} \rrbracket(x)$ is zero at all $x$ which are not jump points.

By (3-17), at points at which $\dot{\gamma}_{t}(x)=0$ a perturbation $\delta \dot{\gamma}(x)$ is arbitrary positive, and therefore $\left(f_{t}(x)+\tau \dot{f}_{t}(x)\right)$ must be nonnegative and $\left.\llbracket \dot{\gamma}_{t}^{\prime}\right](x)$ must be nonpositive. At points at which $\dot{\gamma}_{t}(x)>0$ a perturbation may have any sign, and therefore the same terms must be zero. Then, the sets of KuhnTucker conditions for $f_{t}$

$$
\dot{\gamma}_{t}(x) \geq 0, \quad f_{t}(x)+\tau \dot{f}_{t}(x) \geq 0, \quad\left(f_{t}(x)+\tau \dot{f}_{t}(x)\right) \dot{\gamma}_{t}(x)=0, \quad \text { a.e. } x \in(0, l),
$$

and for the jumps of $\dot{\gamma}_{t}^{\prime}$,

$$
\dot{\gamma}_{t}(x) \geq 0, \quad \llbracket\left[\dot{\gamma}_{t}^{\prime} \rrbracket(x) \leq 0, \quad \llbracket \dot{\gamma}_{t}^{\prime} \rrbracket(x) \dot{\gamma}_{t}(x)=0, \quad \forall x \in(0, l),\right.
$$

follow. At every jump point $x_{o}$ for $\dot{\gamma}_{t}^{\prime}$,

$$
\llbracket \dot{\gamma}_{t}^{\prime} \rrbracket\left(x_{o}\right)=\dot{\gamma}_{t}^{\prime}\left(x_{o}+\right)-\dot{\gamma}_{t}^{\prime}\left(x_{o}-\right)=\lim _{\varepsilon \rightarrow 0^{+}} \frac{\dot{\gamma}_{t}\left(x_{o}+\varepsilon\right)-\dot{\gamma}_{t}\left(x_{o}\right)}{\varepsilon}-\lim _{\varepsilon \rightarrow 0^{-}} \frac{\dot{\gamma}_{t}\left(x_{o}+\varepsilon\right)-\dot{\gamma}_{t}\left(x_{o}\right)}{\varepsilon} .
$$

For $\dot{\gamma}_{t}\left(x_{o}\right)=0$ the right-hand side is nonnegative, and therefore $\llbracket \dot{\gamma}_{t}^{\prime} \rrbracket\left(x_{o}\right)$ is nonnegative. But it is also nonpositive by (3-22) 2 . Hence, a point at which $\dot{\gamma}_{t}\left(x_{o}\right)=0$ cannot be a jump point for $\dot{\gamma}_{t}^{\prime}$. On the other hand, when $\dot{\gamma}_{t}\left(x_{o}\right)$ is positive, $\left[\dot{\gamma}_{t}^{\prime} \rrbracket\left(x_{o}\right)\right.$ is zero by the complementarity condition $(3-22)_{3}$. Then $\llbracket \dot{\gamma}_{t}^{\prime} \rrbracket\left(x_{o}\right)$ is zero in all cases, that is, there is no creation of new jump points for $\dot{\gamma}_{t}^{\prime}$ in the second-order minimization. In particular, if $\gamma_{t}^{\prime}$ is continuous at initial time $t_{0}$, it remains continuous at all subsequent $t$. In what follows, we only consider processes in which $\dot{\gamma}_{t}^{\prime}$ is continuous instead of piecewise continuous.

The assumed continuity of $\dot{\gamma}_{t}^{\prime}$ requires a couple of comments. The first is that it does not imply the continuity of $\gamma_{t}^{\prime \prime}$. Indeed, by Hadamard's condition, this is true only if $d x / d t \neq 0$. That is, a discontinuity of $\gamma_{t}^{\prime \prime}$ is possible, provided that it does not change its position with $t$. The second comment is that the 
continuity of $\gamma_{t}^{\prime \prime}$ has been deduced only for the discretized problem. It need not be preserved in the limit when the time step $\tau$ tends to zero. In fact, in the numerical simulations in Section 4 we will find a situation in which the continuity is not preserved in the limit.

The solutions of (3-21) and (3-22) depend on the time step $\tau$, and do not imply the conditions obtained from the first-order minimization. Specifically, the complementarity condition (3-18) ${ }_{3}$ does not hold in the second-order minimization. Therefore, the solutions for $\tau>0$ are not, in general, directions of steepest descent.

Another necessary condition for a minimum of $J$ at $\dot{\gamma}_{t}$ is that the second variation

$$
\delta^{2} J(\delta \dot{\gamma})=\int_{0}^{l}\left(\theta^{\prime \prime}\left(\gamma_{t}(x)\right) \delta \dot{\gamma}^{2}(x)+w^{\prime \prime}\left(\epsilon_{t}\right) \delta \dot{\bar{\gamma}}^{2}+\alpha \delta \dot{\gamma}^{\prime 2}(x)\right) d x
$$

be nonnegative for all $\delta \dot{\gamma}$ for which the first variation

$$
\delta J\left(\dot{\gamma}_{t}, \delta \dot{\gamma}\right)=\int_{0}^{l}\left(f_{t}(x)+\tau \dot{f}_{t}(x)\right) \delta \dot{\gamma}(x) d x
$$

is zero. For $x$ in the elastic zone, $f_{t}(x)$ is positive, and $\left(f_{t}(x)+\tau \dot{f}_{t}(x)\right)$ is positive for sufficiently small $\tau$. Then, by (3-20) $)_{1}$, for sufficiently small $\tau$ the first variation is zero only for perturbations with $\delta \dot{\gamma}(x)=0$ almost everywhere in the elastic zone.

In the inelastic zone, where $f_{t}(x)=0$ by definition, conditions (3-21) reduce to

$$
\dot{\gamma}_{t}(x) \geq 0, \quad \dot{f}_{t}(x) \geq 0, \quad \dot{f}_{t}(x) \dot{\gamma}_{t}(x)=0, \quad \text { a.e. } x \in \mathscr{g}\left(\beta_{t}, \gamma_{t}\right),
$$

and, by $(3-20)_{1}$, the first variation is zero only if

$$
\dot{f}_{t}(x) \delta \dot{\gamma}(x)=0
$$

almost everywhere in the inelastic zone. In any interval at which $\dot{\gamma}_{t}(x)=0$, we have

$$
\dot{f}_{t}(x)=\theta^{\prime \prime}\left(\gamma_{t}(x)\right) \dot{\gamma}_{t}(x)-\dot{\sigma}_{t}-\alpha \dot{\gamma}_{t}^{\prime \prime}(x)=-\dot{\sigma}_{t} .
$$

Because $\dot{f}_{t}(x) \geq 0$ by $(3-24)_{2}$, such intervals do not exist in a hardening response, $\dot{\sigma}_{t}>0$, and may exist only with $\dot{f}_{t}(x)=0$ in a perfect plastic response or only with $\dot{f}_{t}(x)>0$ in a softening response. Then in a softening response condition (3-25) requires $\delta \dot{\gamma}(x)=0$. Leaving aside the exceptional case of a perfectly plastic response, we conclude that the first variation is zero only if $\delta \dot{\gamma}(x)$ is zero in the elastic zone and at almost all points of the inelastic zone at which $\dot{\gamma}_{t}(x)=0$.

Therefore, it is sufficient to consider perturbations $\delta \dot{\gamma}$ with support in the part of the inelastic zone at which $\dot{\gamma}_{t}>0$. Let $\left(a, a+l_{J}\right)$ be an interval in this region. A necessary condition for a minimum is that the integral

$$
\int_{a}^{a+l_{J}}\left(\theta^{\prime \prime}\left(\gamma_{t}(x)\right) \delta \dot{\gamma}^{2}(x)+\frac{l}{l_{J}} w^{\prime \prime}\left(\epsilon_{t}\right) \delta \dot{\bar{\gamma}}^{2}+\alpha \delta \dot{\gamma}^{\prime 2}(x)\right) d x
$$

be nonnegative for all perturbations $\delta \dot{\gamma}$ with $\delta \dot{\gamma}(a)=\delta \dot{\gamma}\left(a+l_{J}\right)=0$. This corresponds to the nonnegativeness of the smallest eigenvalue $\rho_{1}$ of problem (A.15), with $H$ as in (A.5), and with

$$
a=0, \quad l_{J}=L, \quad \theta^{\prime \prime}\left(\gamma_{t}(x)\right)=\alpha h(x), \quad \frac{l_{J}}{l} w^{\prime \prime}\left(\epsilon_{t}\right)=\alpha \omega, \quad \delta \dot{\gamma}(x)=y(x), \quad \delta \overline{\dot{\gamma}}=\frac{l_{J}}{l} \bar{y} .
$$


The last equality follows from the definitions

$$
\bar{y}=\frac{1}{l_{J}} \int_{0}^{l_{J}} y(x) d x, \quad \delta \overline{\dot{\gamma}}=\frac{1}{l} \int_{0}^{l} \delta \dot{\gamma}(x) d x=\frac{1}{l} \int_{0}^{l_{J}} \delta \dot{\gamma}(x) d x,
$$

which hold for $\delta \dot{\gamma}(x)=y(x)$ with support in $\left(0, l_{J}\right)$. Then the relation between $w^{\prime \prime}\left(\epsilon_{t}\right)$ and $\omega$ comes from the identification

$$
\frac{l}{l_{J}} w^{\prime \prime}\left(\epsilon_{t}\right) \delta \bar{\gamma}^{2}=\alpha \omega \bar{y}^{2}
$$

The upper bound (A.17) for $\rho_{1}$ provides the necessary condition

$$
\alpha \lambda_{o J}^{2}+\theta_{o J}^{\prime \prime} \geq 0
$$

where $\lambda_{o J}$ is the smallest eigenvalue of problem (A.1). It is the solution of (A.7), now rewritten in the form

$$
\frac{l_{J}}{l} \frac{w^{\prime \prime}\left(\epsilon_{t}\right)}{\alpha} l_{J}^{2}=\frac{\lambda_{o J}^{3} l_{J}^{3}}{\lambda_{o_{J}} l_{J}-2 \tan \lambda_{o_{J}} l_{J} / 2}
$$

and $\theta_{o j}^{\prime \prime}$ is as in (3-12), with $y_{o j}$ given by (A.8).

Because the first variation $\delta J\left(\dot{\gamma}_{t}, y_{o J}\right)$ is zero and $J$ is quadratic, one has

$$
J\left(\dot{\gamma}_{t}+c y_{o J}\right)=J\left(\dot{\gamma}_{t}\right)+\frac{1}{2} \tau c^{2} \delta^{2} J\left(\dot{\gamma}_{t}, y_{o J}\right)=J\left(\dot{\gamma}_{t}\right)+\frac{1}{2} \tau c^{2} \rho_{1} \int_{0}^{l} y_{o J}^{2}(x) d x,
$$

for every positive constant $c$. Therefore, $\rho_{1} \geq 0$ is a necessary condition for a minimum of $J$. Moreover, for any other perturbation with support in $\left(0, l_{J}\right)$, since the first variation is nonnegative and $\rho_{1}$ is the smallest eigenvalue,

$$
J\left(\dot{\gamma}_{t}+\delta \dot{\gamma}\right) \geq J\left(\dot{\gamma}_{t}\right)+\frac{1}{2} \tau \delta^{2} J\left(\dot{\gamma}_{t}, \delta \dot{\gamma}\right) \geq J\left(\dot{\gamma}_{t}\right)+\frac{1}{2} \tau \rho_{1} \int_{0}^{l} \delta \dot{\gamma}^{2}(x) d x .
$$

Therefore, $\rho_{1} \geq 0$ is also a sufficient condition for a minimum. The lower bound (A.19) for $\rho_{1}$ provides the explicit sufficient condition

$$
\alpha \lambda_{o J}^{2}+\theta_{\min }^{\prime \prime} \geq 0
$$

with

$$
\theta_{\min }^{\prime \prime}=\inf _{x \in\left(0, l_{J}\right)} \theta^{\prime \prime}\left(\gamma_{t}(x)\right)
$$

For $\rho_{1}$ negative, $J\left(\dot{\gamma}_{t}+c y_{o J}\right)$ can take unlimited negative values. Like in the local model, this event corresponds to brittle fracture. Therefore, $\rho_{1} \geq 0$ is a necessary and sufficient condition for a minimum of $J$, and a negative $\rho_{1}$ corresponds to brittle fracture.

It is of interest to see in which cases the continuations are elastic, $\dot{\gamma}_{t}=0$. From (3-19),

$$
\begin{aligned}
J(\dot{\gamma}) & =\int_{0}^{l}\left(f_{t}(x)-\tau w^{\prime \prime}\left(\epsilon_{t}\right) \dot{\beta}_{t}\right) \dot{\gamma}(x) d x+\frac{1}{2} \tau \int_{0}^{l}\left(\theta^{\prime \prime}\left(\gamma_{t}(x)\right) \dot{\gamma}^{2}(x)+w^{\prime \prime}\left(\epsilon_{t}\right) \overline{\dot{\gamma}}^{2}+\alpha \dot{\gamma}^{\prime 2}(x)\right) d x \\
& \geq \int_{0}^{l}\left(f_{t}(x)-\tau w^{\prime \prime}\left(\epsilon_{t}\right) \dot{\beta}_{t}\right) \dot{\gamma}(x) d x+\frac{1}{2} \tau \alpha \rho_{1} \int_{0}^{l} \dot{\gamma}^{2}(x) d x \geq \int_{0}^{l}\left(c \dot{\gamma}(x)+\frac{1}{2} \tau \alpha \rho_{1} \dot{\gamma}^{2}(x)\right) d x,
\end{aligned}
$$


where

$$
c \doteq f_{\min }-\tau w^{\prime \prime}\left(\epsilon_{t}\right) \dot{\beta}_{t}, \quad f_{\min } \doteq \inf _{x \in(0, l)} f_{t}(x)
$$

Because $J(0)=0, \dot{\gamma}=0$ is a minimizer if the right-hand side of the inequality is nonnegative. In particular, $\dot{\gamma}=0$ is a minimizer if both $\rho_{1}$ and $c$ are nonnegative.

There are two remarkable cases in which $c$ is positive. The first is the case of $\dot{\beta}_{t}<0$. That $\dot{\gamma}_{t}=0$ for $\dot{\beta}_{t}<0$ is the nonlocal version of the property (2-31) of elastic unloading for the local model. The second is the case of a strictly elastic regime, that is, of configurations $\left(\beta_{t}, \gamma_{t}\right)$ for which $f_{\min }>0$. Indeed, in this case $c$ is positive for sufficiently small $\tau$. This is a relaxed version of the condition found in the local model, that there is no increase of inelastic deformation in the elastic regime. Here, the same is true only in a strictly elastic regime.

The foregoing discussion can be summarized as follows:

$$
\begin{array}{lll}
\rho_{1} \geq 0 & \Longleftrightarrow & \text { there are minimizers, } \\
\rho_{1}<0 & \Longleftrightarrow & \text { brittle fracture. } \\
\rho_{1} \geq 0 \text { and } \dot{\beta}_{t}<0 & \Longrightarrow & \dot{\gamma}_{t}=0 \text { is a minimizer, } \\
\rho_{1} \geq 0 \text { and } f_{\min }>0 & \Longrightarrow & \dot{\gamma}_{t}=0 \text { is a minimizer. }
\end{array}
$$

The last two implications tell us that, in a quasistatic evolution, fracture may occur only at loading, $\dot{\beta}_{t} \geq 0$, and in a regime which is not strictly elastic.

To determine the explicit form of a continuation $\dot{\gamma}_{t}$ when $c<0$ is not as easy as it was in the local model. Indeed, in the region at which $\dot{\gamma}_{t}(x)=0$, the introduction of the nonlocal term transforms the incremental equilibrium equation $\dot{\sigma}_{t}=\theta^{\prime \prime}\left(\gamma_{t}(x)\right) \dot{\gamma}_{t}(x)$ into the differential equation

$$
\dot{\sigma}_{t}=\theta^{\prime \prime}\left(\gamma_{t}(x)\right) \dot{\gamma}_{t}(x)-\alpha \dot{\gamma}_{t}^{\prime \prime}(x) \text {. }
$$

This region is not known a priori, since its determination is a part of the solution of the problem. Moreover, only in some special cases is a closed-form solution available. One of them is the onset of the inelastic regime, studied in the next subsection.

3.5. The onset of the inelastic regime. Consider a load process $t \mapsto \beta_{t}$ from the natural configuration $\left(\beta_{t_{0}}, \gamma_{t_{0}}\right)=(0,0)$, with $\dot{\beta}_{t}>0$ for all $t$. At $t=t_{0}$ we have

$$
f_{t_{0}}(x)=\theta^{\prime}\left(\gamma_{t_{0}}(x)\right)-\sigma_{t_{0}}-\alpha \gamma_{t_{0}}^{\prime \prime}(x)=\theta^{\prime}(0)>0,
$$

so that the whole bar is in a strictly elastic regime. By continuity, this regime persists over a finite time interval $\left(t_{0}, t_{c}\right)$. In this interval $\dot{\gamma}_{t}=0$ by (3-29) $)_{4}$, and $\gamma_{t}=0$ because of the initial condition $\gamma_{t_{0}}=0$. The deformation of the bar is homogeneous, with

$$
u^{\prime}(x, t)=\epsilon_{t}=\beta_{t}, \quad \sigma_{t}=w^{\prime}\left(\beta_{t}\right), \quad f_{t}=\theta^{\prime}(0)-w^{\prime}\left(\beta_{t}\right) .
$$

The elastic regime ends at the time $t_{c}$ at which $\beta$ reaches the critical value $\beta_{c}$ given by (2-37). At this time $f_{t}$ becomes zero, and all points of the bar switch from the elastic to the inelastic zone. This is the onset of the inelastic regime. 
To determine the continuation $\dot{\gamma}_{t}$ at $t=t_{c}$, we start from the complementarity condition (3-24) 3 :

$$
0=\dot{f}(x) \dot{\gamma}(x)=\theta^{\prime \prime}(0) \dot{\gamma}^{2}(x)-\dot{\sigma} \dot{\gamma}(x)-\alpha \dot{\gamma}^{\prime \prime}(x) \dot{\gamma}(x) .
$$

Here and in the following, for simplicity, we omit all subscripts $t_{c}$. Integrating over $(0, l)$ and recalling that $\dot{\gamma}^{\prime}$ has no jumps if $\dot{\gamma}$ is a minimizer for $J$, we get

$$
\dot{\sigma} \int_{0}^{l} \dot{\gamma}(x) d x=\int_{0}^{l}\left(\theta^{\prime \prime}(0) \dot{\gamma}^{2}(x)+\alpha \dot{\gamma}^{\prime 2}(x)\right) d x .
$$

If $\theta^{\prime \prime}(0) \geq 0$, the right-hand side is positive and therefore $\dot{\sigma}$ is positive. Moreover, $\dot{\gamma}(x)$ is strictly positive almost everywhere in $(0, l)$. Indeed, if $\dot{\gamma}(x)=0$ in some interval $(a, b)$, from inequality $(3-24)_{2}$ on that interval we have

$$
0 \leq \dot{f}(x)=-\dot{\sigma},
$$

in contradiction with the positiveness of $\dot{\sigma}$. Therefore, $\dot{\gamma}$ is a solution of the differential problem

$$
\theta^{\prime \prime}(0) \dot{\gamma}(x)-\dot{\sigma}-\alpha \dot{\gamma}^{\prime \prime}(x)=0, \quad \forall x \in(0, l), \quad \dot{\gamma}(0)=\dot{\gamma}(l)=0,
$$

subject to the dissipation condition $\dot{\gamma}(x) \geq 0$. For $\theta^{\prime \prime}(0)>0$ the solution is

$$
\dot{\gamma}(x)=\frac{\dot{\sigma}}{\theta^{\prime \prime}(0)}\left(1-\frac{\cosh \kappa(l / 2-x)}{\cosh \kappa l / 2}\right),
$$

with $\kappa=\left(\theta^{\prime \prime}(0) / \alpha\right)^{1 / 2}$. By integration over $(0, l)$,

$$
\overline{\dot{\gamma}}=\frac{\dot{\sigma}}{\theta^{\prime \prime}(0)} \varphi(\kappa l), \quad \varphi(\kappa l) \doteq 1-\frac{\tanh \kappa l / 2}{\kappa l / 2},
$$

and, because $\dot{\sigma}=w^{\prime \prime}\left(\beta_{c}\right)(\dot{\beta}-\overline{\dot{\gamma}})$,

$$
\dot{\sigma}=\frac{\theta^{\prime \prime}(0) w^{\prime \prime}\left(\beta_{c}\right)}{\theta^{\prime \prime}(0)+\varphi(\kappa l) w^{\prime \prime}\left(\beta_{c}\right)} \dot{\beta} .
$$

Comparing with the solution (2-34) of the local model, we see that the nonlocal effect is concentrated in the factor $\varphi(\kappa l)$. Because this factor is positive and less than one, the slope $\dot{\sigma} / \dot{\beta}$ of the response curve $(\sigma, \beta)$ is positive and greater than the slope predicted by the local model, but smaller than the slope $w^{\prime \prime}\left(\beta_{c}\right)$ at unloading.

For $\theta^{\prime \prime}(0)=0$, the solution is

$$
\dot{\gamma}(x)=\frac{\dot{\sigma}}{2 \alpha} x(l-x)
$$

By integration over $(0, l)$,

$$
\overline{\dot{\gamma}}=\frac{\dot{\sigma} l^{2}}{12 \alpha}
$$

and from $\dot{\sigma}=w^{\prime \prime}\left(\beta_{c}\right)(\dot{\beta}-\overline{\dot{\gamma}})$,

$$
\dot{\sigma}=\frac{12 \alpha w^{\prime \prime}\left(\beta_{c}\right)}{12 \alpha+l^{2} w^{\prime \prime}\left(\beta_{c}\right)} \dot{\beta} .
$$

Thus, for $\theta^{\prime \prime}(0)=0$ the perfectly plastic response $\dot{\sigma}=0$ of the local model is replaced by a work-hardening response. 
For $\theta^{\prime \prime}(0)<0$, the solution is

$$
\dot{\gamma}(x)=\frac{w^{\prime \prime}\left(\beta_{c}\right)(\dot{\beta}-\overline{\dot{\gamma}})}{\theta^{\prime \prime}(0)}\left(1-\frac{\cos k(l / 2-x)}{\cos k l / 2}\right),
$$

with $k=\left(-\theta^{\prime \prime}(0) / \alpha\right)^{1 / 2}$. Integrating over $(0, l)$ we find

$$
\overline{\dot{\gamma}}=\frac{w^{\prime \prime}\left(\beta_{c}\right)(\dot{\beta}-\overline{\dot{\gamma}})}{\theta^{\prime \prime}(0)} \psi(k l), \quad \psi(k l)=1-\frac{\tan k l / 2}{k l / 2},
$$

and, therefore,

$$
\overline{\dot{\gamma}}=\frac{\psi(k l) w^{\prime \prime}\left(\beta_{c}\right)}{\theta^{\prime \prime}(0)+\psi(k l) w^{\prime \prime}\left(\beta_{c}\right)} \dot{\beta} .
$$

Again, the difference with (2-33) is due to a single factor, which now is $\psi(k l)$. This factor is negative for $k l<\pi$ and positive for $\pi<k l<2.861 \pi$, with a jump from $-\infty$ to $+\infty$ at $k l=\pi$.

The solution (3-38) satisfies the dissipation inequality (2-10) for all $x$ only if $k l \leq 2 \pi$. Indeed, for $k l>2 \pi, \dot{\gamma}(x)$ takes negative values near the boundary. For such $k l$, let us consider the possibility of localized solutions, that is, of solutions of (3-31) which satisfy the differential equation (3-32) in a subinterval $\left(a, a+l_{i}\right)$ of $(0, l)$ and are zero outside. Without loss of generality, we take the interval $\left(0, l_{i}\right)$, with $l_{i}<l$. The boundary conditions are

$$
\dot{\gamma}(0)=\dot{\gamma}\left(l_{i}\right)=0, \quad \dot{\gamma}^{\prime}\left(l_{i}\right)=0,
$$

where $\dot{\gamma}(0)=0$ is condition (3-15) , and the two remaining conditions are due to the continuity of $\dot{\gamma}$ and $\dot{\gamma}^{\prime}$, which are zero on $\left(l_{i}, l\right)$. The first two conditions determine a solution of the form (3-38) with $l$ replaced by $l_{i}$, and the third determines the length

$$
l_{i}=2 \pi / k
$$

Therefore, the solution is

$$
\dot{\gamma}(x)=\frac{w^{\prime \prime}\left(\beta_{c}\right)(\dot{\beta}-\overline{\dot{\gamma}})}{\theta^{\prime \prime}(0)}(1-\cos k x), \quad x \in\left(0, l_{i}\right) .
$$

It holds for $l_{i}<l$, that is, for $k l>2 \pi$. For $k l=2 \pi$, it coincides with (3-38). By integration over $\left(0, l_{i}\right)$ it follows that

$$
l \overline{\dot{\gamma}}=l_{i} \frac{w^{\prime \prime}\left(\beta_{c}\right)(\dot{\beta}-\overline{\dot{\gamma}})}{\theta^{\prime \prime}(0)}
$$

and, therefore,

$$
\overline{\dot{\gamma}}=\frac{w^{\prime \prime}\left(\beta_{c}\right)}{w^{\prime \prime}\left(\beta_{c}\right)+\theta^{\prime \prime}(0) l / l_{i}} \dot{\beta} .
$$

It is convenient to introduce the positive constant

$$
\psi_{f} \doteq-\frac{\theta^{\prime \prime}(0)}{w^{\prime \prime}\left(\beta_{c}\right)}
$$

With this constant, and with $\psi_{o}$ as in (A.13), (3-40) and (3-44) take the common form

$$
\overline{\dot{\gamma}}=\frac{\psi_{o}(k l)}{\psi_{o}(k l)-\psi_{f}} \dot{\beta} .
$$


For $k l<\pi, \psi_{o}(k l)$ is negative. Therefore, the condition $\overline{\dot{\gamma}} \geq 0$ is satisfied. For $k l>\pi$ the numerator becomes positive, and $\overline{\dot{\gamma}}$ is positive only if

$$
\psi_{o}(k l)>\psi_{f}
$$

From (3-46), using (2-32), we get the incremental response law

$$
\dot{\sigma}=\frac{\theta^{\prime \prime}(0)}{\psi_{o}(k l)-\psi_{f}} \dot{\beta} .
$$

The slope $\dot{\sigma} / \dot{\beta}$ of the response curve is positive if $k l<\pi$, and negative if $k l>\pi$ and condition (3-47) holds. Therefore, at the onset of the inelastic regime, for $\theta^{\prime \prime}(0)$ negative the continuation is

$$
\begin{array}{ll}
\text { work-hardening } & \text { if } k l<\pi, \\
\text { strain-softening } & \text { if } k l>\pi \text { and } \psi_{o}(k l)>\psi_{f} .
\end{array}
$$

In the separating case $k l=\pi$ the continuation is perfectly plastic. For $k l>\pi$ and $\psi_{o}(k l) \leq \psi_{f}$, by (3-47), there is no continuation obeying the dissipation inequality (2-10).

We already know that in the solution (3-40) $\dot{\gamma}(x)$ is zero only at the boundary, while in the solution (3-43) $\dot{\gamma}(x)$ is zero on a portion of $(0, l)$ of positive length $\left(l-l_{i}\right)$. We say that the first is a full-size solution, and that the second is a localized solution. Therefore, for $\theta^{\prime \prime}(0)$ negative, at the onset of the inelastic regime the continuations are

$$
\begin{aligned}
\text { full-size } & \text { if } k l \leq 2 \pi, \\
\text { localized } & \text { if } k l>2 \pi
\end{aligned}
$$

From (3-34) and (3-37) it is clear that for $\theta^{\prime \prime}(0) \geq 0$ all continuations are work-hardening and full-size.

The continuations (3-38) and (3-43) have been determined using the Kuhn-Tucker conditions (3-21) and (3-22), which are necessary for a minimum for the functional $J$ defined in (3-19). It remains to check whether or not these continuations are indeed minimizers. At the onset, $f_{t}$ is zero. Then, neglecting the factor $\tau / 2, J$ has the form

$$
J(\dot{\gamma})=\int_{0}^{l}\left(\theta^{\prime \prime}(0) \dot{\gamma}^{2}(x)+w^{\prime \prime}\left(\beta_{c}\right) \overline{\dot{\gamma}}^{2}+\alpha \dot{\gamma}^{\prime 2}(x)-2 w^{\prime \prime}\left(\beta_{c}\right) \dot{\beta}_{t} \dot{\gamma}(x)\right) d x,
$$

and direct computation yields

$$
J(\dot{\gamma}+\delta \dot{\gamma})=J(\dot{\gamma})+2 \int_{0}^{l} \dot{f}_{t}(x) \delta \dot{\gamma}(x) d x+\int_{0}^{l}\left(\theta^{\prime \prime}(0) \delta \dot{\gamma}^{2}(x)+w^{\prime \prime}\left(\beta_{c}\right) \delta \overline{\dot{\gamma}}^{2}+\alpha \delta \dot{\gamma}^{\prime 2}(x)\right) d x .
$$

On the right side, the first integral is nonnegative by (3-20) $)_{1}$, and the second integral has the form (3-26). Then we can use the results of Section 3.4. In the present case, both constants $\theta_{o_{J}}^{\prime \prime}$ and $\theta_{\min }^{\prime \prime}$ which appear in the bounds (3-27) and (3-28) are equal to $\theta^{\prime \prime}(0)$, and the two bounds coincide. They provide the necessary and sufficient condition $\alpha \lambda_{o}^{2} \geq-\theta^{\prime \prime}(0)$, that is,

$$
\lambda_{o}^{2} \geq k^{2},
$$

where $\lambda_{o}^{2}$ is the smallest eigenvalue of problem (A.1), with $H$ as in (A.5). 
Consider first the case $\pi<k l \leq 2 \pi$, for which the continuation (3-38) is positive over $(0, l)$. In this case, $\lambda_{o}$ is the solution of (A.7) with

$$
L=l, \quad \omega=w^{\prime \prime}\left(\beta_{c}\right) / \alpha, \quad y(x)=\delta \dot{\gamma}(x),
$$

that is,

$$
\frac{w^{\prime \prime}\left(\beta_{c}\right)}{\alpha} l^{2}=\frac{\lambda_{o}^{2} l^{2}}{\psi\left(\lambda_{o} l\right)}
$$

Recalling the definition (3-45) of $\psi_{f}$, we have

$$
\frac{w^{\prime \prime}\left(\beta_{c}\right)}{\alpha}=\frac{-\theta^{\prime \prime}(0)}{\alpha \psi_{f}}=\frac{k^{2}}{\psi_{f}},
$$

and, therefore,

$$
\psi_{f}=\frac{k^{2}}{\lambda_{o}^{2}} \psi\left(\lambda_{o} l\right)
$$

If $k \leq \lambda_{o}$, then

$$
\psi\left(\lambda_{o} l\right) \leq \psi(k l)=\psi_{o}(k l),
$$

because $\psi$ is a decreasing function and, by (A.13), coincides with $\psi_{o}$ for $\pi<k l \leq 2 \pi$. Then, $k \leq \lambda_{o}$ implies inequality (3-47). Conversely, if $k>\lambda_{o}$ the above inequality is reversed. That is, for $\pi<k l \leq 2 \pi$ the condition (3-49) for a minimum coincides with the condition (3-47) for a nonnegative $\overline{\dot{\gamma}}$.

Now consider the case $k l>2 \pi$, for which the continuation (3-43) is positive only on $\left(0, l_{i}\right)$, with $l_{i}=2 \pi / k<l$. In this case, $\lambda_{o}$ is the solution of (A.7) with

$$
L=l_{i}, \quad \omega=\frac{l_{i}}{l} \frac{w^{\prime \prime}\left(\beta_{c}\right)}{\alpha}, \quad y(x)=\delta \dot{\gamma}(x), \quad \bar{y}=\frac{l}{l_{i}} \delta \overline{\dot{\gamma}},
$$

as shown in Section 3.4. Using (3-51), (A.7) takes the form

$$
\frac{l_{i}}{l} \frac{k^{2}}{\psi_{f}} l_{i}^{2}=\frac{\lambda_{o}^{2} l_{i}^{2}}{\psi\left(\lambda_{o} l_{i}\right)} .
$$

Recalling that for $k l>2 \pi$, by (A.13),

$$
\psi_{o}(k l)=\frac{2 \pi}{k l}=\frac{l_{i}}{l}
$$

we finally get

$$
\psi_{f}=\frac{k^{2}}{\lambda_{o}^{2}} \psi_{o}(k l) \psi\left(\lambda_{o} l_{i}\right)
$$

If $k \leq \lambda_{o}$, then $\lambda_{o} l_{i} \geq k l_{i}=2 \pi$. Therefore,

$$
\psi\left(\lambda_{o} l_{i}\right) \leq \psi(2 \pi)=1,
$$

and inequality (3-47) follows. If $k>\lambda_{o}$, then $2 \pi>\lambda_{o} l_{i}$, and the reversed inequality holds. Thus, the coincidence of (3-47) with (3-49) is proved for all $k l>\pi$.

The incremental response law (3-48) shows that, when these inequalities are satisfied as equalities, the slope $\dot{\sigma} / \dot{\beta}$ of the response curve becomes infinite. This is the totally brittle fracture, that is, the 
catastrophic fracture at the onset of the inelastic deformation already described by the local model. The difference is that, while in the local model brittle fracture occurs as soon as $\theta^{\prime \prime}$ ceases to be positive, here it occurs at negative values of $\theta^{\prime \prime}$. Therefore, the sharp inequality (3-47) is a safety condition against totally brittle fracture.

It is commonly asserted that the nonlocal energy term introduces an internal length of the material. Here, this length can be identified with the length $l_{i}$ defined by (3-42), which is the length of the localization zone in a localized continuation. As seen before, the ratio $l / l_{i}=k l / 2 \pi$ determines the quality of the response, hardening or softening, full-size or localized.

The present analysis suggests the definition of a second characteristic length

$$
l_{c}=2 \pi \sqrt{\frac{\alpha}{w^{\prime \prime}\left(\beta_{c}\right)}},
$$

with which the no-fracture condition (3-47) takes the form $\psi_{o}\left(2 \pi l / l_{i}\right)>l_{c}^{2} / l_{i}^{2}$, that is,

$$
l / l_{c}>\frac{l / l_{i}}{\sqrt{\psi_{o}\left(2 \pi l / l_{i}\right)}} \doteq \Psi\left(l / l_{i}\right) .
$$

The parameters which determine the response at the onset are the bar length $l$ and the material constants $w^{\prime \prime}\left(\beta_{c}\right), \theta^{\prime \prime}(0)$, and $\alpha$. With the above definitions, they reduce to the ratios $l / l_{c}$ and $l / l_{i}$. A complete representation of the response is given in Figure 1. In it, each point of the plane represents a bar. The points located below the curve $l / l_{c}=\Psi\left(l / l_{i}\right)$ correspond to bars which undergo totally brittle fracture at the onset of the inelastic deformation. The region above the curve is divided into three subregions,

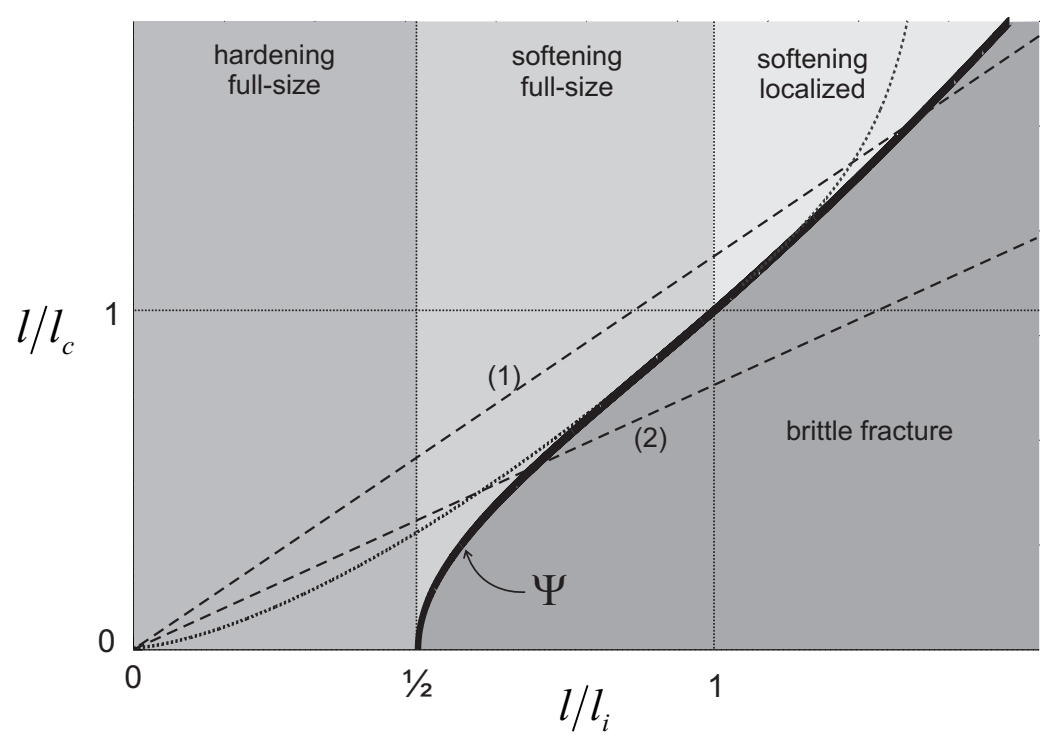

Figure 1. The response at the onset of the inelastic deformation. The three regions above the curve correspond to hardening full-size, softening full-size, and softening localized responses. The region below the curve corresponds to totally brittle fracture. Lines (1) and (2) represent families of bars made of the same material and with varying length. 
corresponding to full-size and hardening $\left(l / l_{i}<1 / 2\right)$, full-size and softening $\left(1 / 2<l / l_{i}<1\right)$, and localized and softening $\left(l / l_{i}>1\right)$ continuations, respectively.

The straight lines from the origin represent families of bars made of the same material and with $l$ growing with the distance from the origin. All families have full-size hardening continuations for small $l$, softening continuations for intermediate $l$, and catastrophic failure for large $l$. Moreover, for families with slope $l_{i} / l_{c}>1$ the softening continuations may be both full-size and localized, while for those with $l_{i} / l_{c}<1$, corresponding to straight lines which do not cross the third region above the curve, the softening continuations can only be full-size.

3.6. Collapse mechanisms. In the nonlocal model there are two possible collapse mechanisms, brittle fracture and ductile fracture. Both are characterized by the slope $\dot{\sigma} / \dot{\beta}$ of the response curve, which is $-\infty$ for brittle fracture and zero for ductile fracture. Brittle fracture coincides with the unique collapse mode provided by the local model. As shown in the next section, the two collapse modes are observed in steel and in concrete, respectively.

In a quasistatic evolution $t \mapsto\left(\beta_{t}, \gamma_{t}\right)$, assume that $\theta^{\prime \prime}\left(\gamma_{t}(x)\right)$ is negative at all $x$, and that it can be approximated by a negative constant $\theta_{t}^{\prime \prime}$. Define the current values of $k$ and $\psi_{f}$ :

$$
k_{t} \doteq \sqrt{\frac{-\theta_{t}^{\prime \prime}}{\alpha}}, \quad \psi_{f t} \doteq \frac{-\theta_{t}^{\prime \prime}}{w^{\prime \prime}\left(\epsilon_{t}\right)} .
$$

With them, the relations (3-46) and (3-48), obtained for the onset of the inelastic regime, can be extrapolated to the subsequent evolution

$$
\overline{\dot{\gamma}}_{t}=\frac{\psi_{o}\left(k_{t} l\right)}{\psi_{o}\left(k_{t} l\right)-\psi_{f t}} \dot{\beta}_{t}, \quad \dot{\sigma}_{t}=\frac{\theta_{t}^{\prime \prime}}{\psi_{o}\left(k_{t} l\right)-\psi_{f t}} \dot{\beta}_{t},
$$

and condition (3-47) against brittle fracture can be replaced by

$$
\psi_{o}\left(k_{t} l\right)>\psi_{f t} .
$$

This extrapolation helps us to understand some general features of the bar's response in the softening regime. Of course, the conclusions are purely qualitative, since the exact values of $\theta_{t}^{\prime \prime}$ and $k_{t}$ are not known. More precise results can only be obtained from numerical simulation.

Assume that $-\theta^{\prime \prime}$ is monotonic increasing. For all $x$, the function $t \mapsto-\theta^{\prime \prime}\left(\gamma_{t}(x)\right)$ is increasing as well, because $t \mapsto \gamma_{t}(x)$ is increasing by the dissipation inequality (2-10). Then the average $-\theta_{t}^{\prime \prime}$ is increasing, $k_{t}$ is increasing, and $\psi_{o}\left(k_{t} l\right)$ is decreasing. Supposing $w^{\prime \prime}\left(\epsilon_{t}\right)$ substantially independent of $t$, $\psi_{f t}$ is increasing. Therefore, the positive denominator in (3-55) decreases with $t$. When it becomes zero, the negative slope of the response curve becomes infinite, and catastrophic failure takes place.

On the contrary, for $-\theta^{\prime \prime}$ monotonic decreasing, $-\theta_{t}^{\prime \prime}$ decreases with $t$, and the slope $\left|\dot{\sigma}_{t} / \dot{\beta}_{t}\right|$ in (3-55) decreases as well. If $-\theta_{t}^{\prime \prime}$ tends to zero, the slope tends to zero, and this corresponds to ductile fracture. Thus, brittle fracture takes place for $-\theta^{\prime \prime}$ increasing, that is, for $\theta^{\prime}$ concave, and ductile fracture takes place for $\theta^{\prime}$ convex. In spite of some early statements and more recent observations, see [Hillerborg 1991] and [Jirásek and Rolshoven 2009b, Figure 16], respectively, this correlation between fracture modes and the concavity-convexity properties of $\theta^{\prime}$ has not received adequate attention in the literature.

This correlation is illustrated by Figure 1. If $\theta_{t}^{\prime \prime}$ increases with $t$, the internal length $l_{i t}=2 \pi / k_{t}$ decreases and the ratio $l / l_{i t}$ increases, while $l / l_{c}$ stays approximately constant. The point representing 
the current status of the bar moves horizontally to the right, tending to brittle fracture which, at the onset, occurs at the curve $\Psi$. On the contrary, if $\theta_{t}^{\prime \prime}$ decreases with $t$ the point moves to the left, tending to the perfectly plastic response which, at the onset, takes place at the line $l / l_{i}=1 / 2$.

The function $\theta^{\prime}$ cannot be concave for all $\gamma>0$. Indeed, concavity implies

$$
\theta^{\prime}(\gamma) \leq \theta^{\prime}(0)+\gamma \theta^{\prime \prime}(0)
$$

and for $\theta^{\prime \prime}(0)<0$ the assumption $\theta^{\prime}(\gamma)>0$ is violated for sufficiently large $\gamma$. On the other hand, the assumption (2-7) $)_{3}$ that $\theta$ has a finite limit at $+\infty$ requires that

$$
\lim _{\gamma \rightarrow+\infty} \theta^{\prime \prime}(\gamma)=0
$$

Then $-\theta^{\prime \prime}$ has a maximum at a finite $\gamma$. Brittle fracture occurs only if this maximum produces a $k_{t}$ sufficiently large to violate inequality (3-56).

\section{Numerical simulations}

For the nonlocal model, quasistatic evolution in the inelastic regime has been investigated by performing a number of numerical simulations. An iterative procedure was used: after fixing a time step $\tau$, for each $t=n \tau$ the continuation $\dot{\gamma}_{t}$ at $\left(\beta_{t}, \gamma_{t}\right)$ was determined approximately by solving a sequence of discretized minimum problems, with the data taken from the solution at $t=(n-1) \tau$.

In the case of loading, $\dot{\beta}>0$, the parameter $t$ is identified with the load $\beta$, so that $\dot{\beta}=1$, and the time step $\tau$ is in fact a load step. The discretized problem consists in minimizing a quadratic approximation of the energy $E^{\mathrm{eq}}\left(\beta_{t}+\tau \dot{\beta}, \gamma_{t}+\tau \dot{\gamma}\right)$. For convenience, we refer to the more general functional

$$
\begin{aligned}
F(\beta, \gamma, \tau, \delta \gamma) & =\int_{0}^{l}\left(\theta(\gamma(x))+w(\beta-\bar{\gamma})+\frac{1}{2} \alpha \gamma^{\prime 2}(x)\right) d x \\
& +\tau \int_{0}^{l}\left(\theta^{\prime}\left(\gamma_{t}(x)\right) \delta \gamma(x)+w^{\prime}(\beta-\bar{\gamma})(1-\delta \gamma(x))+\alpha \gamma^{\prime}(x) \delta \gamma^{\prime}(x)\right) d x \\
& +\frac{1}{2} \tau^{2}\left(\int_{0}^{l}\left(\theta^{\prime \prime}(\gamma(x)) \delta \gamma^{2}(x)+\alpha \delta \gamma^{\prime 2}(x)\right) d x+l w^{\prime \prime}(\beta-\bar{\gamma})(1-\delta \bar{\gamma})^{2}\right),
\end{aligned}
$$

such that $F\left(\beta_{t}, \gamma_{t}, 0,0\right)$ coincides with $E^{\mathrm{eq}}\left(\beta_{t}, \gamma_{t}\right)$, and $F\left(\beta_{t}, \gamma_{t}, \tau, \dot{\gamma}\right)$ coincides with the second-order approximation (2-28) of $E^{\mathrm{eq}}\left(\beta_{t}+\tau \dot{\beta}, \gamma_{t}+\tau \dot{\gamma}\right)$ for the given $\tau$.

4.1. The algorithm. In the solution procedure an iterative algorithm, implemented in an ad hoc finite element code, was used. At each iteration step, for a given initial configuration $\left(\beta_{0}, \gamma_{0}\right)$, with $\gamma_{0}$ a local minimizer for $F\left(\beta_{0}, \cdot, 0,0\right)$, the configuration $\left(\beta_{0}+\tau, \gamma_{0}+\tau \dot{\gamma}_{0}\right)$ is determined by minimizing the function $F\left(\beta_{0}, \gamma_{0}, \tau, \cdot\right)$, constrained by the dissipation inequality (2-10) and by the boundary conditions (3-6). At each load step, the solution accuracy is refined through an iterative procedure acting on the quadratic approximation

$$
F_{2}\left(\beta_{0}, \gamma, \delta \gamma\right) \doteq F\left(\beta_{0}, \gamma, 0,0\right)+\frac{\partial}{\partial(\delta \gamma)} F\left(\beta_{0}, \gamma, 0,0\right) \delta \gamma+\frac{1}{2} \frac{\partial^{2}}{\partial(\delta \gamma)^{2}} F\left(\beta_{0}, \gamma, 0,0\right) \delta \gamma^{2}
$$

of $F\left(\beta_{0}, \gamma, 0, \delta \gamma\right)$. The algorithm runs as follows. 
1. Incremental step.

(i) Compute

$$
\dot{\gamma}_{0}=\operatorname{argmin}\left\{F\left(\beta_{0}, \gamma_{0}, \tau, \cdot\right) \mid \delta \gamma(x) \geq 0, \delta \gamma(0)=\delta \gamma(l)=0\right\} .
$$

(ii) Set $\beta_{1}=\beta_{0}+\tau, \gamma_{1}^{0}=\gamma_{0}+\tau \dot{\gamma}_{0}$.

2. Iterative refinement.

(i) Compute

$$
\delta \gamma^{1}=\operatorname{argmin}\left\{F_{2}\left(\beta_{1}, \gamma_{1}^{0}, \cdot\right) \mid \delta \gamma(x) \geq \gamma_{0}(x)-\gamma_{1}^{0}(x), \delta \gamma(0)=\delta \gamma(l)=0\right\},
$$

and set $\gamma_{1}^{1}=\gamma_{1}^{0}+\delta \gamma^{1}$.

(ii) Compute

$$
\delta \gamma^{i}=\operatorname{argmin}\left\{F_{2}\left(\beta_{1}, \gamma_{1}^{i-1}, \cdot\right) \mid \delta \gamma(x) \geq \gamma_{0}(x)-\gamma_{1}^{i-1}(x), \delta \gamma(0)=\delta \gamma(l)=0\right\},
$$

and set $\gamma_{1}^{i}=\gamma_{1}^{i-1}+\delta \gamma^{i}$.

(iii) Stop when the $L^{2}$ norm of $\left(\gamma_{1}^{i}-\gamma_{1}^{i-1}\right)$ is less than a given tolerance $\hat{\gamma}$.

3. End.

(i) Take as $\gamma_{1}$ the last $\gamma_{1}^{i}$ in the preceding iteration.

(ii) If the $L^{2}$ norm of $\left(\gamma_{1}-\gamma_{0}\right)$ is less than a given tolerance $\tilde{\gamma}$, perform a new incremental step from $\left(\beta_{1}, \gamma_{1}\right)$. Otherwise, repeat the computation with $\tau$ replaced by $\tau / 2$.

The last control avoids the overcoming of energy barriers due to exceedingly large incremental steps. In the numerical code, the spatial variable $x$ is discretized using linear finite elements. The quadratic programming problems involving the minimization of $F_{2}$ are solved using the projection method [Polak 1971; Gill et al. 1981] implemented in the quadprog. m function of MATLAB. The code generates a mesh refinement when the number of elements in the inelastic zone is smaller than a certain number (100 in the simulations presented below). In this case, each element is split into two subelements.

4.2. Choice of energy densities. For the elastic strain energy density we take the quadratic expression

$$
w(\epsilon)=\frac{1}{2} E A \epsilon^{2},
$$

where the axial stiffness $E A$ is the product of the Young modulus $E$ of the material and the area $A$ of the cross-section. For the cohesive energy density we take the piecewise cubic function

$$
\theta(\gamma)=A_{i}+B_{i} \gamma+\frac{1}{2} C_{i} \gamma^{2}+\frac{1}{6} D_{i} \gamma^{3}, \quad \gamma \in\left[\gamma_{i-1}, \gamma_{i}\right],
$$

where

$$
\left\{\left[\gamma_{i-1}, \gamma_{i}\right], i=1, \ldots, n,\right\}
$$


is a subdivision of the interval $\left[\gamma_{0}, \gamma_{n}\right]$ into $n$ adjacent subintervals, with $\gamma_{0}=0$ and $\gamma_{n} \leq+\infty$. The coefficients $A_{i}, B_{i}, C_{i}$ and $D_{i}$ satisfy the $3(n-1)$ conditions

$$
\begin{aligned}
& B_{i+1}=B_{i}-3\left(A_{i+1}-A_{i}\right) \gamma_{i}^{-1}, \\
& C_{i+1}=C_{i}+6\left(A_{i+1}-A_{i}\right) \gamma_{i}^{-2}, \quad i=1, \ldots,(n-1), \\
& D_{i+1}=D_{i}-6\left(A_{i+1}-A_{i}\right) \gamma_{i}^{-3},
\end{aligned}
$$

which guarantee the continuity of $\theta, \theta^{\prime}$, and $\theta^{\prime \prime}$ at $\gamma_{i}$. We fix $A_{1}=\theta(0)=0$, while $B_{1}=\theta^{\prime}(0)$, the value of the axial force at the onset of the inelastic regime, is identified on the experimental curve. The remaining constants are determined by fixing $n+1$ coefficients, for example, $C_{1}, D_{1}$, and $A_{j}$ for $j=2, \ldots, n$. In the following simulations these constants are selected with the purpose of reproducing the response curves of two specific experimental tests, one on a steel bar and one on a concrete specimen.

4.3. Simulation 1: The tensile response of a steel bar. For the first simulation, the data were obtained from a test on a steel bar, stretched to rupture under controlled end displacements. The test was made in Ancona, at the Laboratorio Prove Materiali e Strutture of the Università Politecnica delle Marche. The specimen was a ribbed bar, made of B450C (FeB44k) steel, with diameter $\phi=16 \mathrm{~mm}$ and length $l=200 \mathrm{~mm}$. The axial stiffness

$$
E A=42 \times 10^{3} \mathrm{kN}
$$

is the product of the Young modulus of steel, $E=210 \mathrm{kN} / \mathrm{mm}^{2}$, and the cross-sectional area $A=\pi \phi^{2} / 4$. The axial force at the onset of the inelastic deformation, measured on the response diagram, is

$$
B_{1}=109.5 \mathrm{kN} \text {. }
$$

With the purpose of investigating the size effect, simulations with three different lengths,

$$
l=100,200,300 \mathrm{~mm},
$$

were made. The nonlocality parameter $\alpha$ was taken equal to

$$
\alpha=10 \mathrm{kN} \mathrm{mm}^{2} \text {. }
$$

This is a tentative value. The criteria for choosing the value of $\alpha$ are still under investigation.

For the time step $\tau$, mesh size $h$, and tolerances $\hat{\gamma}$ and $\tilde{\gamma}$ introduced in Section 4.1, the values

$$
\tau=10^{-4}, \quad h=0.5 \mathrm{~mm}, \quad \hat{\gamma}=10^{-6}, \quad \tilde{\gamma}=10^{-3},
$$

were chosen. These values provide good accuracy and, at the same time, keep a moderate size for the discretized problem. For the cohesive energy density a function $\theta_{1}$ of the form (4-3) was taken, with

$$
\begin{aligned}
& n=4, \quad \gamma_{i}=(0.10,1.62,2.01,10), \quad C_{1}=380 \mathrm{kN}, \\
& D_{1}=-3800 \mathrm{kN}, \quad A_{i}=(0,-0.61,499.69,223.74) \mathrm{kN} .
\end{aligned}
$$

This energy, plotted at the top in Figure 2, is convex for $\gamma<0.1$ and concave for $\gamma>0.1$.

In Figure 3, the experimental response curve (the dotted line) is compared with the response curves of simulations with lengths $l=100,200,300 \mathrm{~mm}$. In the experimental curve, the initial hardening regime is followed by a softening regime, in which the negative slope of the curve increases with $\beta$, and becomes 


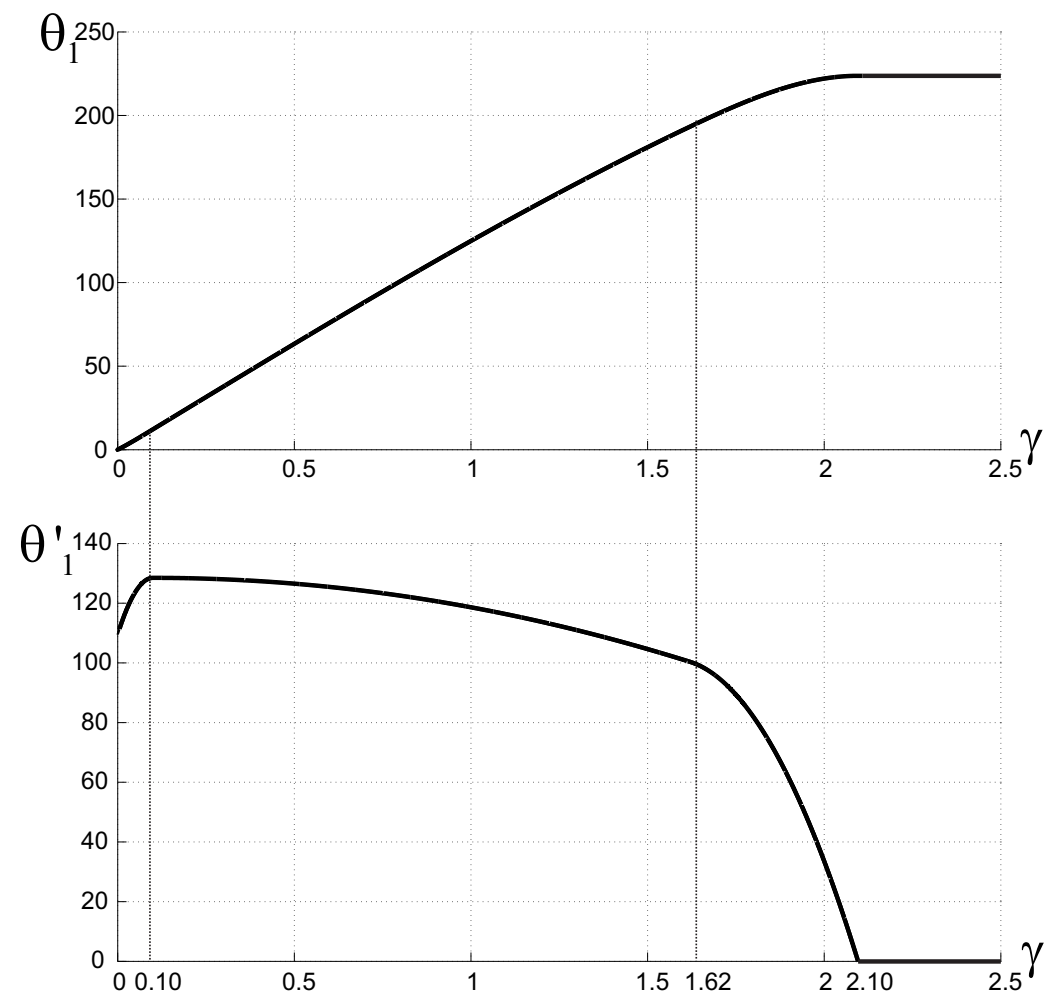

Figure 2. Simulation 1: the cohesive energy $\theta_{1}$ (top) and its derivative $\theta_{1}^{\prime}$ (bottom).

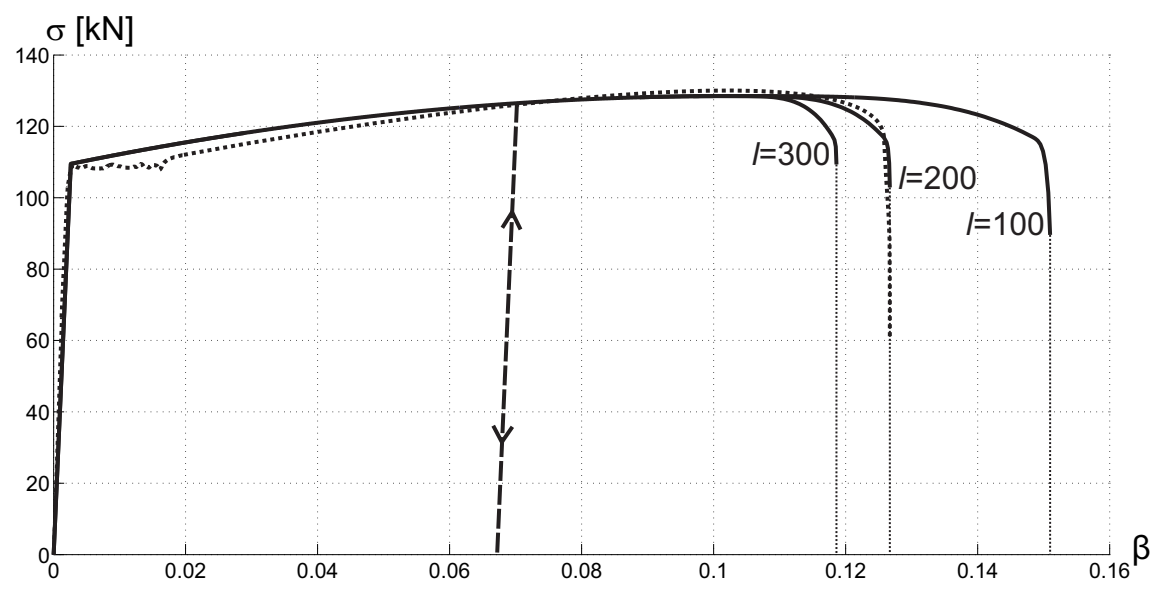

Figure 3. Simulation 1: the experimental response curve (dotted line), and the response curves of simulations with different values of $l$. The dashed line shows the model's response to unloading and reloading.

infinite at rupture. In the model, this behavior is reproduced by taking $\theta_{1}$ initially convex, and then concave with growing concavity. 

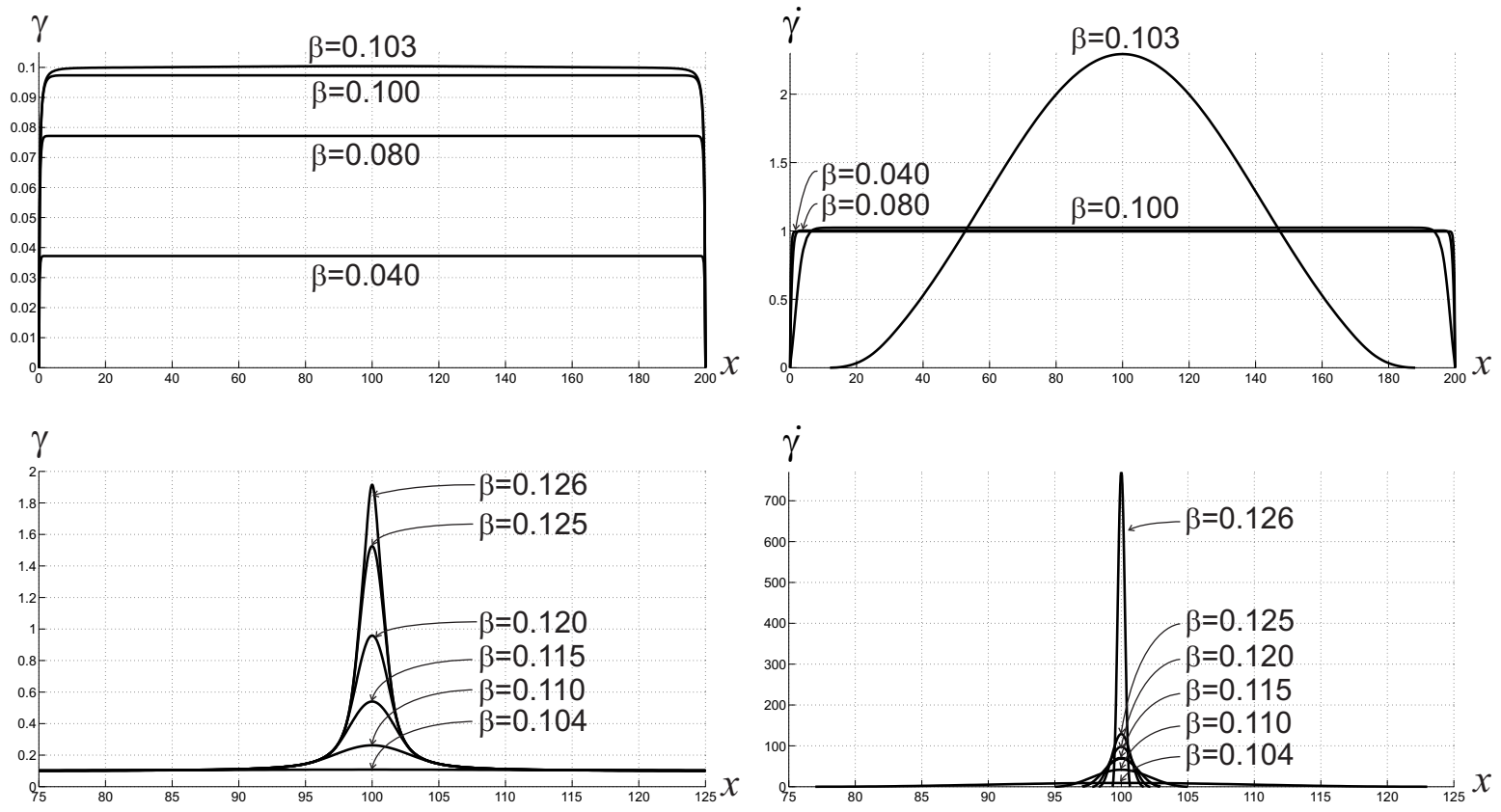

Figure 4. Simulation 1 with $l=200 \mathrm{~mm}$. Evolution of $\gamma$ (left column) and $\dot{\gamma}$ (right column) for increasing $\beta$.

The response curve of the simulation with $l=200 \mathrm{~mm}$, which is the specimen's length, is very close to the experimental curve. Of course, this is due to an appropriate choice of the material constants. Anyway, it is remarkable that a small number of constants is sufficient to reproduce the evolution of the bar's deformation from the natural configuration to final rupture. Also remarkable is the perfect reproduction of the elastic unloading, shown by the dashed line in the figure, and obtained by reversing the sign of $\dot{\beta}$ up to complete unloading, $\sigma=0$, and then reloading. As shown in the figure, the reloading curve traces back the unloading line and then continues along the loading curve of monotonic loading.

In the three simulations shown in Figure 3, the hardening parts of the response curves are identical. The hardening regime ends at $\beta \approx 0.102$, where the force $\sigma$ reaches its maximum and a softening regime starts. Shortly after, strain localization begins, and the three curves separate. The figure shows that for longer bars the softening regime produces larger negative slopes, and rupture occurs at smaller $\beta$. This size effect agrees with the theoretical preview $(3-55)_{2}$.

The evolution of $\gamma$ and $\dot{\gamma}$ in the simulation with $l=200 \mathrm{~mm}$ is shown in Figure 4. In the initial hardening regime, represented in the upper part of the figure, both $\gamma$ and $\dot{\gamma}$ are constant, except near the boundary, where $\gamma(x)=\dot{\gamma}(x)=0$ as required by the boundary conditions.

The regime of full-size softening response is very short. Indeed, it starts at $\beta \approx 0.102$ and ends at $\beta \approx 0.103$ when, as shown in the two right panels in the figure, localization takes place. For larger $\beta$, the deformation concentrates at shorter and shorter central zones of the bar. The left half of Figure 5 reproduces the curves of Figure 4, bottom left; note the close similarity of these diagrams with the results of experiments made long ago by Miklowitz [1950], shown on the right in Figure 5. Also, the evolution curves for $\gamma$ are qualitatively the same as the curves in [Jirásek and Rolshoven 2009b, Figure 17.1]. 

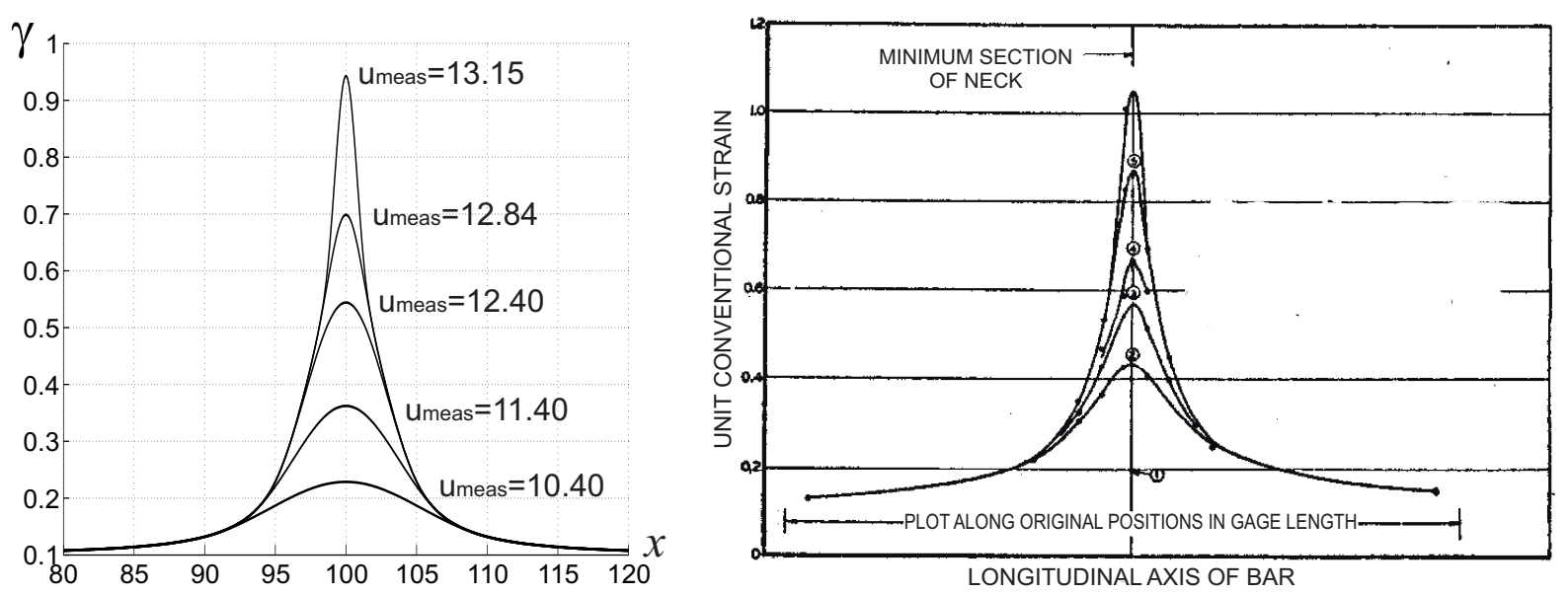

Figure 5. Left: detail of Figure 4, bottom left. Right: experiments on a cylindrical bar of medium-carbon steel, taken from [Miklowitz 1950].

4.4. Simulation 2: The tensile response of a concrete specimen. The aim of our second simulation was to reproduce the experimental force-elongation curve of a prismatic specimen made of lightweight concrete, taken from [Hordijik 1992, Figure 38]. The specimen is prismatic, with dimensions of $50 \times 50 \times$ $150 \mathrm{~mm}$, Young's modulus $E=18 \mathrm{kN} / \mathrm{mm}^{2}$, and maximum aggregate size of $8 \mathrm{~mm}$. In the simulation we take

$$
E A=45 \times 10^{3} \mathrm{kN}, \quad B_{1}=6.3 \mathrm{kN},
$$

with $B_{1}$ the axial force at the onset of the inelastic regime, read on the experimental curve. We consider the three lengths

$$
l=100,150,200 \mathrm{~mm} .
$$

For the nonlocality parameter $\alpha$ we take

$$
\alpha=3497 \mathrm{kN} \mathrm{mm}^{2} \text {. }
$$

This value corresponds to an internal length $l_{i}=2 \pi / k=8 \mathrm{~mm}$, equal to the maximum aggregate size. For the time step $\tau$, mesh size $h$, and tolerances $\hat{\gamma}$ and $\tilde{\gamma}$ we choose

$$
\tau=10^{-7}, \quad h=0.25 \mathrm{~mm}, \quad \hat{\gamma}=10^{-6}, \quad \tilde{\gamma}=10^{-3} .
$$

For the cohesive energy we take the function $\theta_{2}$ represented in Figure 6. This is an energy of the form (4-3), with

$$
n=4, \quad \gamma_{i}=(0.7,1.2,65.0,100.0) \times 10^{-4},
$$

and with

$$
C_{1}=18 \times 10^{3} \mathrm{kN}, \quad D_{1}=-25.7143 \times 10^{7} \mathrm{kN}, \quad A_{i}=(0,-0.12,-0.25,154.27) \times 10^{-4} \mathrm{kN} .
$$

This energy is convex for $\gamma<0.7 \times 10^{-4}$ and concave for $\gamma>0.7 \times 10^{-4}$.

The force-elongation response curves for the three considered values of $l$ are shown in Figure 7. The correspondence with the experimental curve is good, but not as good as in the preceding simulation. 

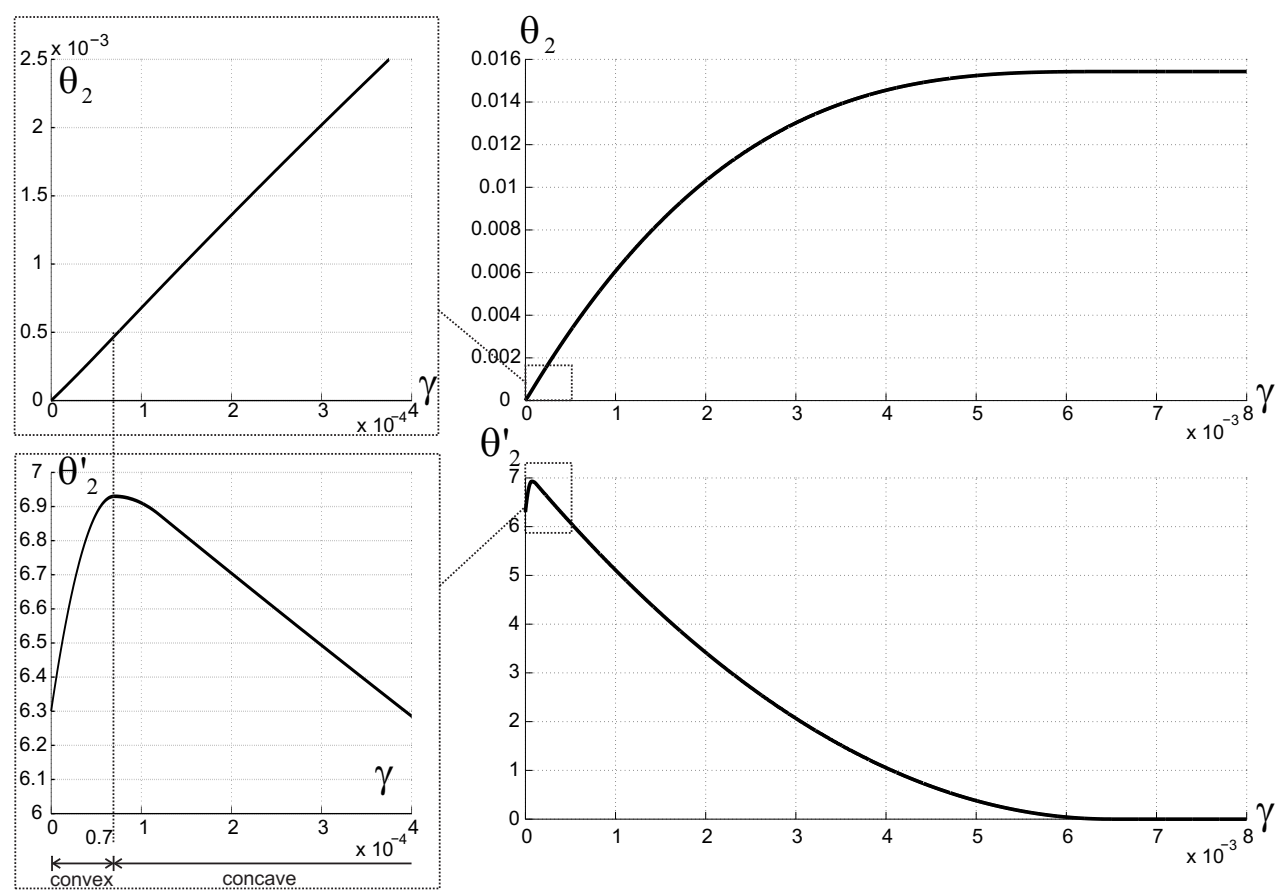

Figure 6. Simulation 2: the cohesive energy $\theta_{2}$ (top) and its derivative $\theta_{2}^{\prime}$ (bottom).

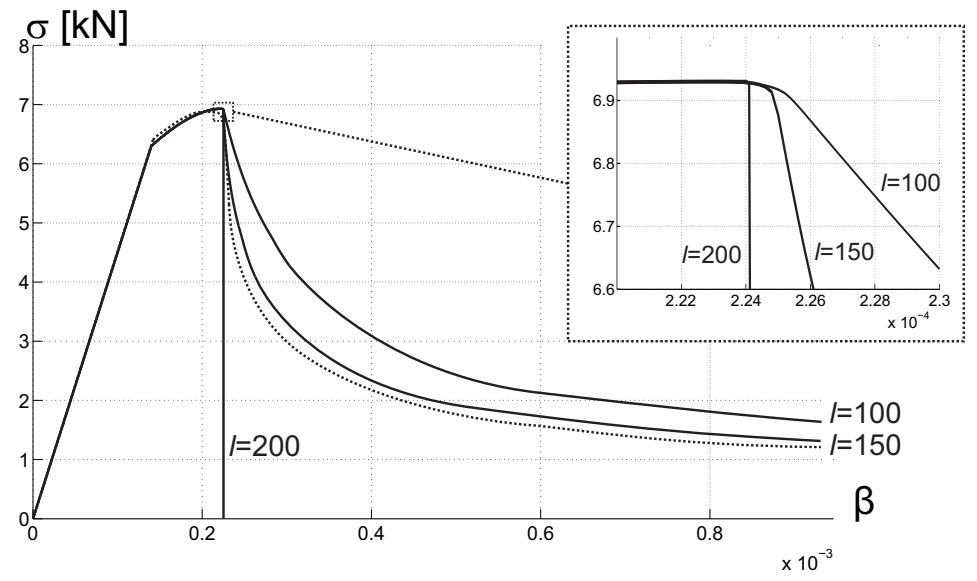

Figure 7. Simulation 2: the experimental (dotted line) and the numerical response curves (solid line).

This may be due to the fact that in the experimental curve in [Hordijik 1992] the elongation $\beta_{m} l_{m}$ was measured on a central zone of the bar, of length $l_{m}=35 \mathrm{~mm}$, while here the total elongation was evaluated with the approximate formula

$$
\beta l \approx \beta_{m} l_{m}+\left(l-l_{m}\right)(\beta-\bar{\gamma}),
$$

obtained by neglecting the inelastic deformation outside the central zone. 
Another cause of discrepancy is that in the experiment the specimen had a central notch, which was not considered in the simulation. Generally, the data fitting was more difficult than in Simulation 1, where all data came from our own experiment and not from one taken from the literature.

As in the preceding simulation, the three numerical curves coincide in the elastic regime and in the subsequent hardening and full-size softening regimes. They start to differ at the onset of localization, at $\beta \approx 2.23 \times 10^{-4}$. In the localized softening regime, in accordance with the size effect, the negative slope of the response curve is larger for longer bars. The curve for $l=200 \mathrm{~mm}$ exhibits brittle fracture at the onset of localization. This agrees with the safety condition (3-56) against brittle fracture, which now takes the form

$$
\frac{2 \pi}{k_{t} l}>\frac{\alpha k_{t}^{2}}{E A}
$$

Indeed, in a localized solution $\psi_{o}\left(k_{t} l\right)$ is equal to $2 \pi / k_{t} l$ by (A.13), and $\psi_{f t}$ is equal to $\alpha k_{t}^{2} / E A$ by (3-54) and (4-2). Setting $k_{t}=2 \pi / l_{t}$, with $l_{t}=8 \mathrm{~mm}$ equal to the maximum aggregate size, condition (3-56) becomes

$$
l<\frac{2 \pi E A}{k_{t}^{3} \alpha}=\frac{E A l_{t}^{3}}{4 \pi^{2} \alpha} \approx 167 \mathrm{~mm} .
$$

For the bars with $l=100 \mathrm{~mm}$ and $150 \mathrm{~mm}$, the convex shape of $\theta_{2}^{\prime}$ determines a ductile fracture mechanism. For $l=150 \mathrm{~mm}$, as shown in Figure 8 , at $\beta \approx 2.22 \times 10^{-4}$ the inelastic strain rate $\dot{\gamma}$ begins to localize and the force starts to decrease. The length of the localized zone attains a minimum of about $8 \mathrm{~mm}$ at $\beta \approx 2.25 \times 10^{-4}$, and then steadily increases. Numerical investigations not reported here
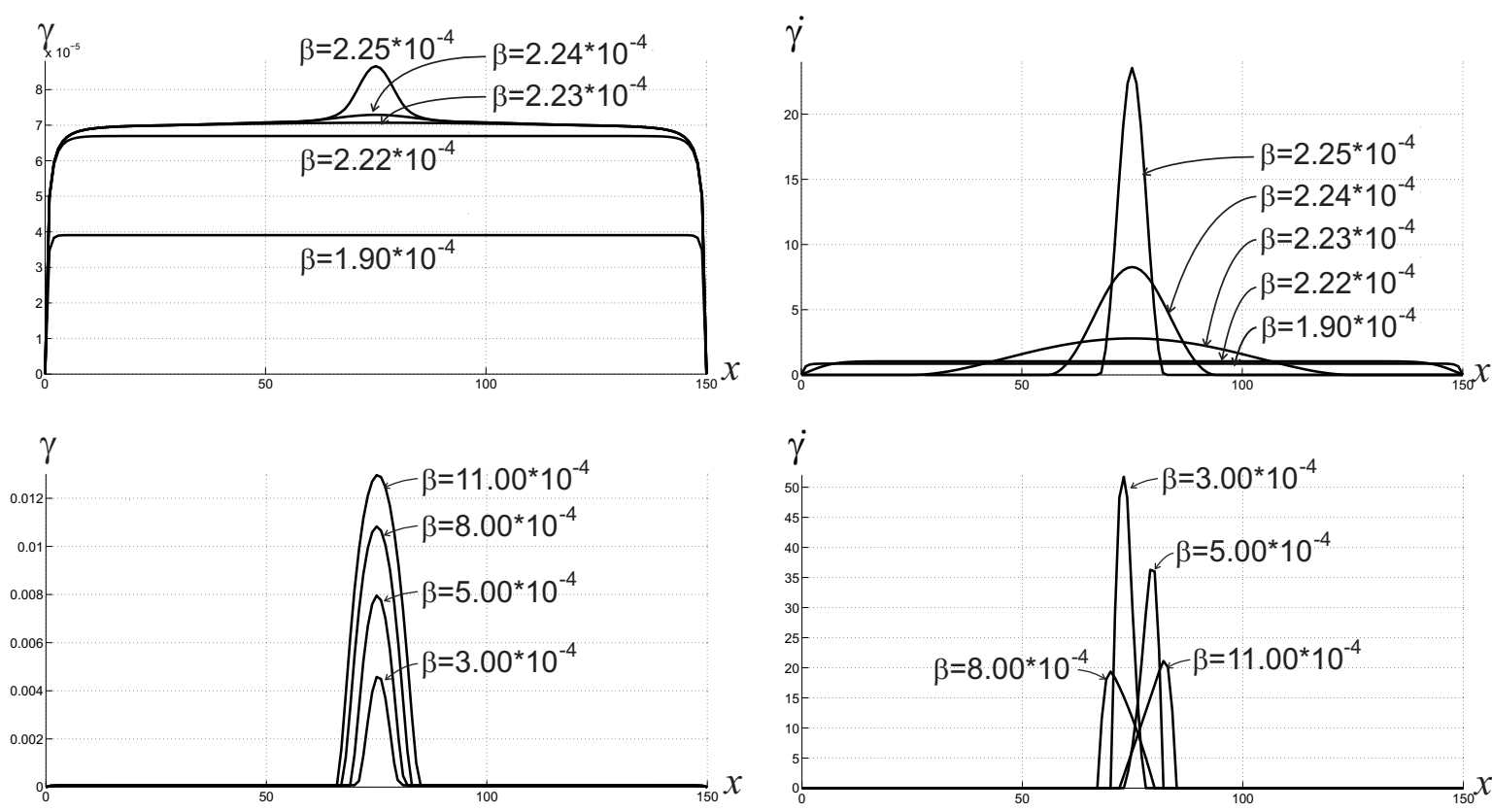

Figure 8. Simulation 2: evolution of the inelastic deformation for $l=150 \mathrm{~mm}$. 
show that the localization zone eventually diffuses over the whole bar. For the bar with $l=200 \mathrm{~mm}$, catastrophic failure occurs at $\beta \approx 2.24 \times 10^{-4}$.

The evolution of $\gamma$ and $\dot{\gamma}$ is shown in Figure 8. The asymmetries of the diagrams of $\dot{\gamma}$ in the bottom right panel of Figure 8 are purely numerical, and random in nature. This is confirmed by the symmetry of their time integral $\gamma$ in the bottom left panel. For $\beta \approx 10^{-3}$ the inelastic deformation $\gamma$ is concentrated on a portion of the bar of length of about $20 \mathrm{~mm}$. This agrees with an estimate given in [Bažant and Pijaudier-Cabot 1989], according to which the characteristic length of concrete is about 2.7 times the maximum aggregate size.

By effect of the expansion of the inelastic zone the force decreases, and tends to a horizontal asymptote. In [Jirásek 1998; Jirásek and Rolshoven 2009a], the expansion of the inelastic zone is considered as unphysical, and the stress locking, that is, the convergence of the force to a positive limit, is considered as a proof of this.

While the horizontal asymptotes of the response curve shown in [Jirásek 1998, Figure 3], are about $80 \%$ of the maximum $\sigma$, a numerical investigation, not reported here, shows that in the present simulation the horizontal asymptote is only about $2 \%$ of the maximum $\sigma$. This modest residual force cannot be eliminated. Indeed, looking at the diagrams of $\gamma$ in the bottom left panel of Figure 8 we see that, for $\beta>4.5 \times 10^{-4}$, the largest value $\gamma(l / 2)$ of $\gamma$ is greater than $6 \times 10^{-3}$. Therefore, as shown by the constitutive curve of Figure 6, bottom, the corresponding value of $\theta^{\prime}$ is zero. Because the point $x=l / 2$ is in the inelastic zone, from the yield condition (3-2) with $\theta^{\prime}=0$ it follows that

$$
\sigma=-\alpha \gamma^{\prime \prime}(l / 2)
$$

with $\gamma^{\prime \prime}(l / 2)$ strictly negative because $\gamma$ has a maximum at $x=l / 2$.

\section{Appendix: Solution of some eigenvalue problems}

Consider the eigenvalue problem

$$
\int_{0}^{L}\left(y^{\prime 2}(x)+\omega \bar{y}^{2}\right) d x=\lambda^{2} \int_{0}^{L} y^{2}(x) d x, \quad y \in H,
$$

with $\omega$ a given positive constant, and

$$
\bar{y}=\frac{1}{L} \int_{0}^{L} y(x) d x .
$$

The eigenfunctions are the solutions of the Euler equation

$$
y^{\prime \prime}(x)-\omega \bar{y}+\lambda^{2} y(x)=0,
$$

and have the form

$$
y(x)=A \sin \lambda x+B \cos \lambda x+\omega \lambda^{-2} \bar{y} .
$$

We wish to determine the smallest eigenvalue $\lambda_{o}^{2}$ and the corresponding eigenfunctions $y_{o}$, for two different choices of the domain $H$.

(i) Let $H$ be the set

$$
H=\left\{y \in H^{1}(0, L) \mid y(0)=y(L)=0\right\} .
$$


In this case, the constants $A$ and $B$ are

$$
A=-\omega \lambda^{-2} \bar{y} \tan \lambda L / 2, \quad B=-\omega \lambda^{-2} \bar{y} .
$$

Moreover, integrating (A.4) over $(0, L)$ we get

$$
L \bar{y}=\omega \lambda^{-3} \bar{y}((\cos \lambda L-1) \tan \lambda L / 2-\sin \lambda L+\lambda L)=\omega \lambda^{-3} \bar{y}(\lambda L-2 \tan \lambda L / 2),
$$

that is,

$$
\omega L^{2}=\frac{\lambda^{3} L^{3}}{\lambda L-2 \tan \lambda L / 2}=\frac{\lambda^{2} L^{2}}{\psi(\lambda L)},
$$

with $\psi$ as in (3-39) 2 . The right-hand side is an increasing function of $\lambda L$, and is equal to zero at $\lambda L=\pi$ and to $+\infty$ at $\lambda L \approx 2.861 \pi$. Therefore, for every $\omega L^{2}>0$ there exists a unique $\lambda L$ in $(\pi, 2.861 \pi)$ which satisfies (A.7). It determines the smallest eigenvalue $\lambda_{o}^{2}$ for given $\omega$ and $L$. The corresponding eigenfunctions are the scalar multiples of

$$
y_{o}(x)=\cos \lambda_{o}(L / 2-x)-\cos \lambda_{o} L / 2 .
$$

The problem has been formulated in $H^{1}(0, L)$, which is the largest space in which the integrals which appear in (A.1) exist. However, the eigenfunction $y_{o}$ belongs to $C^{\infty}[0, L]$. So the solution does not change if $H$ is taken to be any space included between $C^{\infty}[0, L]$ and $H^{1}(0, L)$.

(ii) Now let $H$ be the set

$$
H=\left\{y \in H^{2}(0, L) \mid y(0)=y(L)=0, y(x) \geq 0, \forall x \in(0, L)\right\} .
$$

In (A.8), $y_{o}$ satisfies condition $y(x) \geq 0$ only for $\lambda_{o} L \leq 2 \pi$. By (A.7), this corresponds to $\omega L^{2} \leq 4 \pi^{2}$. For larger values of $\omega L^{2}, y_{o}(x)$ becomes negative near the boundary.

For $\omega L^{2}>4 \pi^{2}$ we look for solutions $y$ which are of the form (A.4) in a subinterval $\left(0, L_{y}\right)$ of $(0, L)$ and zero outside. To belong to $H^{2}(0, L)$, they must satisfy the conditions

$$
y(0)=y\left(L_{y}\right)=0, \quad y^{\prime}\left(L_{y}\right)=0 .
$$

By (A.4), the first two conditions are satisfied by

$$
A=-\omega \lambda^{-2} \bar{y} \tan \lambda L_{y} / 2, \quad B=-\omega \lambda^{-2} \bar{y},
$$

and the third condition is satisfied if $A=0$. This implies $\tan \lambda L_{y} / 2=0$, that is,

$$
\lambda L_{y}=2 \pi
$$

Because $L_{y}$ is smaller than $L$, this solution is possible only for $\lambda L>2 \pi$, that is, for $\omega L^{2}>4 \pi^{2}$. But this is exactly the range not covered by the solution (A.7) and (A.8). For $A=0$ and $B=-\omega \lambda^{-2} \bar{y}$, the integration of (A.4) yields

$$
\omega L^{2}=\frac{\lambda^{2} L^{3}}{L_{y}}=\frac{\lambda^{3} L^{3}}{2 \pi} .
$$


Therefore, for all $\omega L^{2}>4 \pi^{2}$ the smallest eigenvalue is the solution $\lambda_{o}^{2}$ of this equation. The corresponding eigenfunction

$$
y_{o}(x)= \begin{cases}1-\cos \lambda_{o} x, & \text { if } x \leq 2 \pi / \lambda_{o}, \\ 0, & \text { if } x>2 \pi / \lambda_{o}\end{cases}
$$

satisfies the condition $y_{o}(x) \geq 0$ for all $x$. Therefore, $\lambda_{o}^{2}$ is the smallest eigenvalue, and $y_{o}$ is a corresponding eigenfunction.

In conclusion, the smallest eigenvalue of problem (A.1) with $H$ as in (A.9) is the solution of (A.7) if $\omega L^{2} \leq 4 \pi^{2}$, and of (A.11) if $\omega L^{2}>4 \pi^{2}$. By defining

$$
\psi_{o}(\lambda L) \doteq \begin{cases}\psi(\lambda L), & \text { if } 0 \leq \lambda L \leq 2 \pi, \\ \frac{2 \pi}{\lambda L}, & \text { if } 2 \pi<\lambda L,\end{cases}
$$

both (A.7) and (A.11) take the form

$$
\lambda^{2}=\omega \psi_{o}(\lambda L)
$$

and the smallest eigenvalue $\lambda_{o}^{2}$ is the unique solution of this equation. The function $\psi_{o}$ is $C^{\infty}$ in $(\pi,+\infty)$, except at the point $\lambda L=2 \pi$, where it is continuous and has a continuous derivative.

Now consider the eigenvalue problem

$$
\int_{0}^{L}\left(y^{\prime 2}(x)+\omega \bar{y}^{2}+h(x) y^{2}(x)\right) d x=\rho \int_{0}^{L} y^{2}(x) d x, \quad y \in H,
$$

with $\omega$ a given positive constant and $h$ an integrable function. We wish to determine upper and lower bounds for the smallest eigenvalue $\rho_{o}$, both for $H$ as in (A.5) and as in (A.9).

For $H$ as in (A.5), let $\lambda_{o}^{2}$ be the smallest eigenvalue of problem (A.1) and let $y_{o}$ be a corresponding eigenfunction. Define

$$
h_{o} \doteq \frac{\int_{0}^{L} h(x) y_{o}^{2}(x) d x}{\int_{0}^{L} y_{o}^{2}(x) d x} .
$$

Then, by (A.1),

$$
\int_{0}^{L}\left(y_{o}^{\prime 2}(x)+\omega \bar{y}_{o}^{2}+h_{o} y_{o}^{2}(x)\right) d x=\left(\lambda_{o}^{2}+h_{o}\right) \int_{0}^{L} y_{o}^{2}(x) d x .
$$

That is, $\left(\lambda_{o}^{2}+h_{o}\right)$ is an eigenvalue of (A.15) and $y_{o}$ is a corresponding eigenfunction. Therefore, an upper bound for $\rho_{o}$ is

$$
\lambda_{o}^{2}+h_{o} \geq \rho_{o}
$$

To get a lower bound, set

$$
h_{\min }=\inf _{x \in(0, L)} h(x)
$$


Then the left-hand side of (A.15) is greater than or equal to

$$
\int_{0}^{L}\left(y^{\prime 2}(x)+\omega \bar{y}^{2}+h_{\min } y^{2}(x)\right) d x \geq\left(\lambda_{o}^{2}+h_{\min }\right) \int_{0}^{L} y^{2}(x) d x, \quad \forall y \in H,
$$

and the lower bound

$$
\rho_{o} \geq \lambda_{o}^{2}+h_{\min }
$$

follows. Depending on the given function $h$, the two bounds can be far away from each other. On the other hand, they coincide if $h$ is a constant, $h(x)=\bar{h}$. Indeed, in this case $h_{o}=h_{\min }=\bar{h}$ and, therefore,

$$
\rho_{o}=\lambda_{o}^{2}+\bar{h}
$$

For $H$ as in (A.9) the same bounds hold, with $\lambda_{o}^{2}$ and $y_{o}$ replaced by the smallest eigenvalue of problem (A.1) and by a corresponding eigenfunction, respectively.

\section{References}

[Aifantis 1984] E. C. Aifantis, "On the microstructural origin of certain inelastic models", J. of Engng. Materials and Technology, ASME 106 (1984), 326-330.

[Aifantis 1992] E. C. Aifantis, "On the role of gradients in the localization of deformation and fracture", Int. J. Engng. Sci. 30 (1992), 1279-1299.

[Ambrosio and Tortorelli 1990] L. Ambrosio and V. M. Tortorelli, "Approximation of functionals depending on jumps by elliptic functionals via $\Gamma$-convergence”, Comm. Pure Appl. Math. 43:8 (1990), 999-1036.

[Babadjian 2011] J.-F. Babadjian, "A quasistatic evolution model for the interaction between fracture and damage", Arch. Ration. Mech. Anal. 200:3 (2011), 945-1002.

[Barenblatt 1962] G. I. Barenblatt, "The mathematical theory of equilibrium cracks in brittle fracture", pp. 55-129 in Advances in applied mechanics, vol. 7, Academic Press, New York, 1962.

[Bažant and Chen 1997] Z. P. Bažant and E. P. Chen, "Scaling of structural failure", Appl. Mech. Rev. 50 (1997), 593-627.

[Bažant and Jirásek 2002] Z. P. Bažant and M. Jirásek, "Nonlocal integral formulations of plasticity and damage: survey of progress", ASCE J. of Engng. Mechanics 128 (2002), 1119-1149.

[Bažant and Le 2009] Z. P. Bažant and J. L. Le, "Size effect on strength and lifetime distributions of quasibrittle structures", Proc. ASME Int. Mech. Engng. Congress IMECE (2009), 1-9.

[Bažant and Pijaudier-Cabot 1989] Z. P. Bažant and G. Pijaudier-Cabot, "Measurement of characteristic length of nonlocal continuum", ASCE J. of Engng. Mechanics 115 (1989), 755-767.

[Benallal and Marigo 2007] A. Benallal and J.-J. Marigo, "Bifurcation and stability issues in gradient theories with softening", Modelling and Simulation in Materials Science and Engineering 15 (2007), S283-S295.

[Borst and Pamin 1996] R. D. Borst and J. Pamin, "Gradient plasticity in numerical simulation of concrete cracking”, Eur. J. Mech. A/15 (1996), 295-320.

[Bourdin et al. 2000] B. Bourdin, G. A. Francfort, and J.-J. Marigo, "Numerical experiments in revisited brittle fracture", $J$. Mech. Phys. Solids 48:4 (2000), 797-826.

[Cahn and Hilliard 1958] J. W. Cahn and J. E. Hilliard, "Free energy of a nonuniform system, I: Interfacial free energy", J. Chem. Physics 28 (1958), 258-267.

[Carpinteri 1982] A. Carpinteri, “Application of fracture mechanics to concrete structures”, ASCE J. Struct. Division 108 (1982), 833-848.

[Carpinteri and Massabò 1997] A. Carpinteri and R. Massabò, "Continuous vs discontinuous bridged-crack model for fiberreinforced materials in flexure”, Int. J. Solids Structures 34 (1997), 2321-2338.

[Casal 1972] P. Casal, "La théorie du second gradient et la capillarité”, C. R. Acad. Sc. Paris 274 (1972), A1571-1574. 
[Dal Maso and Toader 2002] G. Dal Maso and R. Toader, "A model for the quasi-static growth of brittle fractures: existence and approximation results", Arch. Ration. Mech. Anal. 162:2 (2002), 101-135.

[Dal Maso and Toader 2010] G. Dal Maso and R. Toader, "Quasistatic crack growth in elasto-plastic materials: the twodimensional case", Arch. Ration. Mech. Anal. 196:3 (2010), 867-906.

[Del Piero and Truskinovsky 2009] G. Del Piero and L. Truskinovsky, "Elastic bars with cohesive energy", Contin. Mech. Thermodyn. 21:2 (2009), 141-171.

[Del Piero et al. 2012] G. Del Piero, G. Lancioni, and R. March, "Diffuse cohesive energy in plasticity and fracture", Technische Mechanik 32 (2012), 174-188.

[Drucker 1952] D. C. Drucker, "A more fundamental approach to plastic stress-strain relations", pp. 487-491 in Proceedings of the First U. S. National Congress of Applied Mechanics (Chicago, 1951), The American Society of Mechanical Engineers, New York, 1952.

[Ericksen 1990] J. L. Ericksen, "Liquid crystals with variable degree of orientation", Arch. Rational Mech. Anal. 113:2 (1990), 97-120.

[Fleck and Hutchinson 2001] N. A. Fleck and J. W. Hutchinson, "A reformulation of strain gradient plasticity", J. Mech. Phys. Solids 49 (2001), 2245-2271.

[Francfort and Marigo 1998] G. A. Francfort and J.-J. Marigo, "Revisiting brittle fracture as an energy minimization problem", J. Mech. Phys. Solids 46:8 (1998), 1319-1342.

[Frank 1958] F. C. Frank, "On the theory of liquid crystals", Discuss. Faraday Soc. 25 (1958), 19-28.

[Freddi and Royer-Carfagni 2010] F. Freddi and G. Royer-Carfagni, "Regularized variational theories of fracture: a unified approach", J. Mech. Phys. Solids 58:8 (2010), 1154-1174.

[Gill et al. 1981] P. E. Gill, W. Murray, and M. H. Wright, Practical optimization, Academic Press [Harcourt Brace Jovanovich Publishers], London, 1981.

[Griffith 1920] A. A. Griffith, "The phenomena of rupture and flow in solids", Phil. Trans. Roy. Soc. A221 (1920), 163-198.

[Gudmundson 2004] P. Gudmundson, "A unified treatment of strain gradient plasticity”, J. Mech. Phys. Solids 52:6 (2004), $1379-1406$.

[Gurtin 2003] M. E. Gurtin, "On a framework for small-deformation viscoplasticity: free energy, microforces, strain gradients", Int. J. of Plasticity 19 (2003), 47-90.

[Gurtin and Anand 2005] M. E. Gurtin and L. Anand, "A theory of strain-gradient plasticity for isotropic, plastically irrotational materials, I: Small deformations", J. Mech. Phys. Solids 53:7 (2005), 1624-1649.

[Hill 1950] R. Hill, The Mathematical Theory of Plasticity, Oxford, at the Clarendon Press, 1950.

[Hillerborg 1991] A. Hillerborg, "Application of the fictitious crack model to different types of materials", Int. J. of Fracture 51 (1991), 95-102.

[Hillerborg et al. 1976] A. Hillerborg, M. Modéer, and P. Peterson, "Analysis of crack formation and crack growth in concrete by means of fracture mechanics and finite elements", Cement and Concrete Research 6 (1976), 773-782.

[Hordijik 1992] D. A. Hordijik, "Tensile and tensile fatigue behaviour of concrete: experiments, modelling and analyses", Heron 37 (1992), 1-79.

[Jirásek 1998] M. Jirásek, "Nonlocal models for damage and fracture: comparison of approaches", Int. J. Solids Structures 35:31-32 (1998), 4133-4145.

[Jirásek and Rolshoven 2009a] M. Jirásek and S. Rolshoven, "Localization properties of strain-softening gradient plasticity models, I: Strain-gradient theories", Int. J. Solids Structures 46 (2009), 2225-2238.

[Jirásek and Rolshoven 2009b] M. Jirásek and S. Rolshoven, "Localization properties of strain-softening gradient plasticity models, II: Theories with gradients of internal variables", Int. J. Solids Structures 46 (2009), 2239-2254.

[Marigo and Truskinovsky 2004] J.-J. Marigo and L. Truskinovsky, "Initiation and propagation of fracture in the models of Griffith and Barenblatt", Contin. Mech. Thermodyn. 16:4 (2004), 391-409.

[Miklowitz 1950] J. Miklowitz, "The influence of the dimensional factors on the mode of yielding and fracture in medium-carbon steel. II. The size of the round tensile bar", J. Appl. Mech 17 (1950), 159-168. 
[Mumford and Shah 1989] D. Mumford and J. Shah, "Optimal approximations by piecewise smooth functions and associated variational problems", Comm. Pure Appl. Math. 42:5 (1989), 577-685.

[Pham and Marigo 2010a] K. Pham and J.-J. Marigo, “Approche variationnelle de l'endommagement, I: Les concepts fondamentaux", C. R. Mécanique 338 (2010), 191-198.

[Pham and Marigo 2010b] K. Pham and J.-J. Marigo, "Approche variationnelle de l'endommagement, II: Les modèles à gradient", C. R. Mécanique 338 (2010), 199-206.

[Pham et al. 2011] K. Pham, H. Amor, J.-J. Marigo, and C. Maurini, "Gradient damage models and their use to approximate brittle fracture", Int. J. Damage Mechanics 20 (2011), 618-652.

[Polak 1971] E. Polak, Computational methods in optimization: A unified approach, Mathematics in Science and Engineering 77, Academic Press, New York, 1971.

[Truskinovsky 1996] L. Truskinovsky, "Fracture as a phase transition", in Contemporary Research in the Mechanics and Mathematics of Materials, edited by R. C. Batra and M. F. Beatty, Barcelona, 1996.

[Volokh 2004] K. Y. Volokh, "Nonlinear elasticity for modeling fracture of isotropic brittle solids", J. Appl. Mech. 71 (2004), $141-143$.

[van der Waals 1893] J. D. van der Waals, "Thermodynamische theorie der capillariteit in de onderstelling van continue dichtheidsverandering", Verh. Kon. Akad. Weten. (1) 33:8 (1893), 1-56. Translated in J. Stat. Phys. 20 (1979), 197-244, DOI 10.1007/BF01011514.

[Yalcinkaya et al. 2011] T. Yalcinkaya, W. A. M. Brekelmans, and M. G. D. Geers, "Deformation patterning driven by rate dependent non-convex strain gradient plasticity", J. Mech. Phys. Solids 59:1 (2011), 1-17.

Received 6 Jul 2012. Revised 28 Mar 2013. Accepted 10 Apr 2013.

GianPIETRo Del Piero: dlpgpt@unife.it

Dipartimento di Ingegneria, Università di Ferrara, Via Saragat 1, 44100 Ferrara, Italy

GIOVANNI LANCIONI: g. lancioni@univpm.it

Dipartimento di Ingegneria Civile, Edile e Architettura, Università Politecnica delle Marche, Via Brecce Bianche 12, 60131 Ancona, Italy

RICCARDO MARCH: r.march@iac.cnr.it

Istituto per le Applicazioni del Calcolo "Mauro Picone", Consiglio Nazionale delle Ricerche, Via dei Taurini 19, 00185 Roma, Italy 


\title{
JOURNAL OF MECHANICS OF MATERIALS AND STRUCTURES
}

\author{
msp.org/jomms
}

\author{
Founded by Charles R. Steele and Marie-Louise Steele
}

EDITORIAL BOARD

ADAIR R. AGUIAR

KATIA BERTOLDI

University of São Paulo at São Carlos, Brazil

DAVIDE BIGONI

Harvard University, USA

IWONA JASIUK

University of Trento, Italy

Thomas J. PENCE

University of Illinois at Urbana-Champaign, USA

YASUHIDE SHINDO

Michigan State University, USA

DAVID STEIGMANN

Tohoku University, Japan

University of California at Berkeley

\section{ADVISORY BOARD}

J. P. CARTER University of Sydney, Australia

R. M. Christensen Stanford University, USA

G. M. L. GLADWELL University of Waterloo, Canada

D. H. Hodges Georgia Institute of Technology, USA

J. HUTCHINSON Harvard University, USA

C. HwU National Cheng Kung University, Taiwan

B. L. KARIHALOO University of Wales, UK

Y. Y. KIM Seoul National University, Republic of Korea

Z. Mroz Academy of Science, Poland

D. PAMPlonA Universidade Católica do Rio de Janeiro, Brazil

M. B. RubiN Technion, Haifa, Israel

A. N. SHUPIKOV Ukrainian Academy of Sciences, Ukraine

T. TARNAI University Budapest, Hungary

F. Y. M. WAN University of California, Irvine, USA

P. WRIGGERS Universität Hannover, Germany

W. YANG Tsinghua University, China

F. ZIEGLER Technische Universität Wien, Austria

PRODUCTION production@msp.org

SILVIO LEVY Scientific Editor

Cover photo: Mando Gomez, www.mandolux.com

See msp.org/jomms for submission guidelines.

JoMMS (ISSN 1559-3959) at Mathematical Sciences Publishers, 798 Evans Hall \#6840, c/o University of California, Berkeley, CA 94720-3840, is published in 10 issues a year. The subscription price for 2013 is US \$555/year for the electronic version, and $\$ 705 /$ year (+\$60, if shipping outside the US) for print and electronic. Subscriptions, requests for back issues, and changes of address should be sent to MSP.

JoMMS peer-review and production is managed by EditFLOW ${ }^{\circledR}$ from Mathematical Sciences Publishers.

PUBLISHED BY

mathematical sciences publishers

nonprofit scientific publishing

http://msp.org/

(C) 2013 Mathematical Sciences Publishers 


\section{Journal of Mechanics of Materials and Structures}

\section{Volume 8, No.:2-4 April-June 2013}

A diffuse cohesive energy approach to fracture and plasticity: the one-dimensional case

Gianpietro Del Piero, Giovanni Lancioni and Riccardo March 109

Continuum deployable shells made of thin plates

Vladimir A. GracheV and Yuriy S. NeUSTAdT

Self-folding of a slender microbeam and thin film: an elastica model

JIANLIN LIU and JUNG HoON LEE 169

Dispersion of guided waves in initially stressed layered plates

JIANGONG YU and SHENLEI LI 185

Empirical models for predicting protective properties of concrete shields against

high-speed impact

GABI BEN-Dor, ANATOLY DUBinsky and Tov ELPERIN

Transient response of a general anisotropic solid to dislocation growth: alternative formulation LOUIS MILTON BROCK 